\title{
Effect of 8-week n-3 fatty-acid supplementation on oxidative stress and inflammation in middle- and long-distance running athletes: a pilot study
}

Daniela Buonocore $^{1 *}$ (D), Manuela Verri ${ }^{1}$, Andrea Giolitto $^{1}$, Enrico Doria ${ }^{1}$, Michele Ghitti $^{2}$ and Maurizia Dossena ${ }^{1}$

\begin{abstract}
Background: Long-chain n-3 polyunsaturated fatty acids, such as eicosapentaenoic acid (EPA) and docosahexaenoic acid (DHA), may alter oxidative status and immune function after exercise. The aim of this pilot study was to determine the probable association between $\mathrm{n}-3$ supplementation and physical exercise, observing the variations in markers of oxidative stress and inflammation.

Methods: Thirty-nine subjects of both sexes aged 17-30 years were divided into two groups: 1) ( $n=21)$ trained Athletes; 2$)(n=18)$ Sedentary subjects. All subjects were given about $4 \mathrm{~g} /$ day of $n-3$ supplementation, rich in EPA and DHA, for 8 weeks. Blood, saliva and urine samples were collected pre- (TO) and post- (T1) supplementation. Hematological parameters (tryglicerides, total cholesterol, HDL, CPK, LDH, HGH, IGF-1), oxidative markers (MDA, 8OHdG, PCc), antioxidant parameters (GPx, SOD, CAT, DPPH scavenger), exercise-induced stress markers (testosterone and cortisol) and an inflammatory marker (TNF-a) were measured. All tests were two-sided and a $p$-value of less than 0.05 was considered as statistically significant.

Results: The results showed that MDA and TNF-amean values significantly decreased after supplementation in both Athletes and Sedentary subjects: variation was greater in Athletes than in Sedentary control subjects. Generally, our results suggested that supplementation with n-3 PUFAs created a synergic variation in the parameters from a baseline state (T0) to a treated state after supplementation (T1), in terms of size and modality, which was significantly different in Athletes compared to Sedentary subjects.

Conclusion: In conclusion, supplementation with about $4 \mathrm{~g} /$ day of n-3 PUFAs, rich in EPA and DHA, for 8 weeks, seemed to be effective in counteracting some parameters involved in oxidative stress and inflammation, induced by acute strenuous physical exercise.
\end{abstract}

Keywords: Docosahexaenoic acid, Eicosapentaenoic acid, Inflammation, Long-chain n-3 polyunsaturated fatty acids, Nutraceuticals, Oxidative status, Physical exercise

\footnotetext{
* Correspondence: daniela.buonocore@unipv.it

'Department of Biology and Biotechnology "Lazzaro Spallanzani", University of Pavia, Via Ferrata, 9-27100 Pavia (PV), Italy

Full list of author information is available at the end of the article
}

(C) The Author(s). 2020 Open Access This article is licensed under a Creative Commons Attribution 4.0 International License, which permits use, sharing, adaptation, distribution and reproduction in any medium or format, as long as you give appropriate credit to the original author(s) and the source, provide a link to the Creative Commons licence, and indicate if changes were made. The images or other third party material in this article are included in the article's Creative Commons licence, unless indicated otherwise in a credit line to the material. If material is not included in the article's Creative Commons licence and your intended use is not permitted by statutory regulation or exceeds the permitted use, you will need to obtain permission directly from the copyright holder. To view a copy of this licence, visit http://creativecommons.org/licenses/by/4.0/ The Creative Commons Public Domain Dedication waiver (http://creativecommons.org/publicdomain/zero/1.0/) applies to the data made available in this article, unless otherwise stated in a credit line to the data. 


\section{Background}

Physical training and training sessions for middle- and long-distance running, performed by agonistic/sports professional athletes, are examples of intense and prolonged physical exertion. During the period of physical preparation and training sessions, as well as during competition, two fundamental qualities are important in order for an athlete to stand out: speed and endurance, which are closely related to endurance sport performance and to the maximal lactate steady state (MLSS) [1]. Referring to performance, it is well recognized that exhaustive exercise can lead to muscle fatigue, delayed-onset muscle soreness, and a decrement in performance; particularly, the acute strenuous prolonged exercise that is performed during middle- and longdistance running is combined with an increase in reactive oxygen species (ROS) production (including free radicals), changes in blood antioxidant status, and an increase in inflammatory responses. All these events may result in oxidative stress or generally cause DNA damage, lipid peroxidation with increased levels of toxic aldehydes, fatigue, reduction of heme-iron blood levels and release of proinflammatory cytokines (interleukins) [2-5].

Therefore, oxidative stress and inflammation induced by acute strenuous physical exercise should be limited in order for athletes to maintain a state of well-being and health, as they are constantly exposed to stress conditions and to the risk of overreaching, which is considered as an accumulation of training load, leading to performance decrements that require days to weeks for recovery. Moreover, systemic inflammation and subsequent effects on the central nervous system may cause a worsening of this syndrome, known as overtraining syndrome (OTS), including depressed mood, central fatigue, and resultant neuro-hormonal changes [6].

The use of food supplements has been considered as a valid method of reducing oxidative stress and systemic inflammation [7]. Omega-3 or n-3 polyunsatured fatty acids (PUFAs), such as the eicosapentaenoic acid (EPA) and docosahexaenoic acid (DHA), are nutrients that seem to possess both antioxidant and anti-inflammatory effects, primarily through their effects on the neutrophil and macrophage components [8] and on mediators of acute inflammation, such as D-series resolvins, protectins and maresins $[9,10]$. EPA and DHA can cause dual inhibition of cyclo-oxygenase and lipoxygenase pathways for metabolism of arachidonic acid (AA) [11]. Regarding the molecular mechanism, it is known that n-3 PUFAs affect the inflammatory cytochine gene expression, modifying the activity of the transcription nuclear factor - B and the peroxisome proliferator-activated receptor (PPAR- $\gamma$ ) [12].

It has been suggested that the ingestion of $1-2 \mathrm{~g} /$ day of EPA and DHA, at a ratio of 2:1 EPA to DHA, may be beneficial for athletes' health by neutralizing exerciseinduced inflammation [13]. However, the human data are inconclusive as to whether n-3 PUFA supplementation at this dosage is effective in attenuating the inflammatory and immunomodulatory response to exercise $[14,15]$. Some authors do not find merit in such a recommendation, concluding that $\mathrm{n}-3$ PUFA supplementation might be a potential aid, i.e. athletes with exercise-induced bronchoconstriction [16], and other groups may benefit (i.e. strength athletes), but there is insufficient data from high quality studies in this area [17]. Therefore, the main aim of the present longitudinal, prospective, pilot study was to determine the association between long-chain $n-3$ PUFA supplementation, rich in EPA and DHA, and physical exercise, observing the probable variations of markers of lipid profile, muscle workload, oxidative and antioxidant status, steroid hormones as markers of exerciseinduced stress, and inflammatory status in middle- and long-distance running Athletes. It has been hypothesized that EPA/DHA would attenuate the exercise-induced rise in biomarkers, compared to a control group of sedentary subjects. As far as we know, this study is the first of its kind in this sport specialization. The second aim was to study whether a synergistic effect exists for the interaction between two stimuli, physical exercise and n-3 supplementation, on the aforementioned variables.

\section{Materials and methods Subjects}

Thirty-nine healthy subjects of both sexes aged $17-30$ years (average $23.80 \pm 5.88$ ) volunteered to participate in this study. All of the subjects resided in Italy and all but two of them (from north Africa) were Italian with a Mediterranean diet. They were divided into the following two groups:

1) $(n=21)$ Trained Athletes, middle-distance runners ( $800 \mathrm{~m}, 1500 \mathrm{~m}, 3000 \mathrm{~m}$ steeplechase) and long-distance runners $(5000 \mathrm{~m}, 10,000 \mathrm{~m}$, marathon), whose training program included at least $5 \mathrm{~h}$ of physical training a day, every day;

2) $(n=18)$ Sedentary subjects who performed less than one hour of physical activity twice a week.

The following criteria defined the size and expected number of the experimental group: qualitative response model (baseline improvement yes/no); two parallel groups of 22 subjects each. Five subjects (one Athlete and four Sedentary) who were initially recruited for the trial were excluded from the final analysis because they withdrew before the end of the project.

All subjects were fully informed about the experimental protocols. The investigations were carried out following the 1975 Declaration of Helsinki guidelines (https://www.wma.net/what-we-do/medical-ethics/declaration-of-helsinki/), which were revised in Tokyo in 2004 and subsequently in 2013. All subjects approved and signed an informed consent document, authorizing the experimenter to use the results for scientific 
publication purposes; moreover, they each completed a four-day food diary. The Institutional Review Board at the University of Pavia approved the study. None of the subjects had taken any supplements or medications for 4 weeks prior to or during the experiment. All subjects were non-smokers, and none had taken any medication or drugs that could affect the results. The Athletes were all distance runners and cross-country track and field, who take part in national and international competitions. The inclusion criterion of Sedentary subjects was that they performed physical activity no more than twice a week, for a maximum of one hour each time.

\section{Materials}

All chemicals and solvents used in this study were purchased from Sigma-Aldrich, St. Louis, MO, USA and J.T. Baker, USA. The DNA/RNA oxidative damage ELISA kit and the TNF- $\alpha$ ELISA kit were purchased from Cayman Chemical Company, Michigan, USA. Steroid Hormones ELISA kits were purchased from Diametra Diagnostic, Segrate-Milan, Italy. Software MetaDieta ${ }^{\circ}$ for anthropometric and dietary/bromatological data was provided by METEDA srl. San Benedetto del Tronto (AP) Italy/EU. The blood samples were carried out at a Center of Clinical Biochemical Analysis, authorized by the National Health System (Pavia, Italy/EU).

Food Supplements (n-3) were soft-gel capsules containing fish oil (e.g. sardines, anchovies and mackerel, caught in open waters of the southern Pacific Ocean, Chile) at a minimum oil concentration of $40 \%$ EPA and $20 \%$ DHA in the form of ethyl esters. Each capsule of n$3(1380 \mathrm{mg})$ contained $950 \mathrm{mg}$ (>95\%) of fatty acids $\mathrm{n}-3$ ethyl ester (EE), of which $400 \mathrm{mg}$ EPA (43\%) and $200 \mathrm{mg}$ DHA (28\%), and $2.5 \mathrm{mg}$ of vitamin E (2500 ppm). The product was certified to be of high quality and it was free from doping substances with testosterone and nandrolone precursors, and from Beta2 agonists, diuretics, amphetamines and ephedrine.

\section{Experimental design}

Before starting supplementation, anthropometric data (sex, age, weight, height, Body Mass Index-BMI) (Table 1) and a 4-day food diary were collected for each subject. All volunteer subjects participating in the study were instructed by a dietitian to fill in a food diary for 4 nonconsecutive days to evaluate energy intake and consumption of each nutrient, using the software MetaDieta ${ }^{\circ}$ (METEDA S.r.l. - San Benedetto del Tronto, AP, Italy/ $E U)$. We focused on the intake values of $n-6$ and $n-3$ PUFAs and their ratio, as the dramatic change in eating habits in recent years has led to an imbalance in the ratio in favor of n-6 PUFAs, particularly in the Western diet [18]. This change has coincided with a worldwide increase in the incidence of inflammatory bowel disease (IBD) [19].
Table 1 Anthropometric data

\begin{tabular}{|c|c|c|c|c|}
\hline & Age (years) & Weight (kg) & Height (m) & BMI $\left(\mathrm{kg} / \mathrm{m}^{2}\right)$ \\
\hline \multicolumn{5}{|c|}{ ATHLETE } \\
\hline Mean & 25.56 & 60.06 & 1.74 & 19.87 \\
\hline $\mathrm{SD}$ & 6.99 & 7.25 & 0.07 & 1.10 \\
\hline \multicolumn{5}{|c|}{ SEDENTARY } \\
\hline Mean & 22.86 & 57.86 & 1.70 & 19.85 \\
\hline SD & 1.57 & 8.34 & 0.10 & 1.51 \\
\hline
\end{tabular}

Anthropometric data for Athlete (n 21) and Sedentary (n 18) subjects Data are expressed as mean values \pm SD

Some of the anti-inflammatory effects of n-3 PUFAs may be mediated by competition with n-6 PUFAs, because n-3 PUFAs act as a competitive substrate for the metabolism of n-6 PUFAs [20]. The data obtained by the software MetaDieta ${ }^{\circ}$ were compared with the Dietary Reference Values (DRVs), particularly the Reference Intake range for macronutrients (RI), defined by Dietary Reference Intake for the Italian Population for specific ages: IV Revisione dei Livelli di Assunzione di Riferimento di Nutrienti ed energia per la popolazione italiana (LARN), guidelines containing information related to recommendations issued by Società Italiana di Nutrizione Umana (SINU) [21]. These values and recommendations were formulated according to the opinions of the European Food Safety Authority (EFSA) Panel on Dietetic Products, Nutrition and Allergies (NDA),published in 2010, which addressed the general principles for deriving and applying DRVs, an umbrella term for the complete set of nutrient reference values, including population reference intakes (PRIs), the average requirements (ARs), adequate intakes (AIs) and reference intake (RIs) ranges for macronutrients. These values indicate the amount of a nutrient which must be consumed on a regular basis to maintain health in an otherwise healthy individual (or population), referring to the European population [22]. In North America, the World Health Organization (WHO) recommends RI values of $6-10 \%$ of total energy for PUFA intakes for adults [23]. In the United States of America, The National Academies of Science Engineering Medicine recommends the following AI values: $n-6,17 \mathrm{~g} /$ day for young men and $12 \mathrm{~g} /$ day for young women; $\mathrm{n}-3,1.6$ and $1.1 \mathrm{~g} /$ day for men and women, respectively [24]. In Italy, the LARN recommends RI values of $4-8 \%$ of total energy from diet for $n-6$ PUFA and $0.5-2 \%$ of total energy from diet for n-3 PUFA for adults [21].

For Athletes, the number of hours of daily training and the type of sport that were used to calculate energy consumption were also taken into consideration for this study.

The combination of all the above-mentioned parameters led us to the choice of an appropriate amount of daily supplementation: each subject took $4 \mathrm{cps} /$ day of $\mathrm{n}$ 3 , i.e. about $4 \mathrm{~g}$ of $\mathrm{n}-3$ fatty acids. 
All subjects from both groups (Athletes and Sedentary) took $4 \mathrm{cps} /$ day of n-3 (2 at breakfast, 1 at lunch and 1 at dinner) for a period of 8 weeks. Compliance to the dosing regimen was monitored with the capsule count method: subjects received a fixed number of capsules and were asked to return any unused ones at their next follow-up visit.

In order to observe the association between long-chain n3 PUFA supplementation, rich in EPA and DHA, and physical exercise, the variations in different markers of muscle workload, oxidative stress, steroid hormones as markers of exercise-induced stress, and inflammation, in Athletes and Sedentary subjects, were adopted as primary outcomes.

Our secondary outcome was to establish if a synergism existed between two stimuli: physical exercise and n-3 supplementation.

One sample of venous blood (red blood cells/RBC and plasma samples), one sample of saliva and one sample of urine were collected from all subjects at the following time points:

- before food supplementation (time T0) to evaluate different screening parameters;

- at the end of food supplementation (time T1), after 8 weeks ( \pm 5 days), to evaluate the variations in all parameters.

The biological samples were collected in the morning, taking into account the circadian rhythm of the biochemical and physiological parameters, particularly of cortisol and testosterone. Blood samples taken from the antecubital vein were collected in BD Vacutainers Tubes (Vacu$\mathrm{Lab}^{\circ}$ EDTA tubes). Samples were centrifuged (1000 x g for $10 \mathrm{~min}$ at $25^{\circ} \mathrm{C}$ using centrifuge J6-MC by Beckman), and the resultant plasma was aliquoted and stored at $-80^{\circ} \mathrm{C}$. Urine samples were collected in sterile tubes and stored at $-80^{\circ} \mathrm{C}$. Saliva samples were collected in Salivette ${ }^{\circ}$ (No. 51.1534 .500 , Sarsted). Samples were centrifuged (1000 x g for $2 \mathrm{~min}$ at $25^{\circ} \mathrm{C}$ using centrifuge J6-MC by Beckman) to obtain a clear saliva sample ready to be stored at $-80^{\circ} \mathrm{C}$. All samples were analyzed in the same analytical session for each test using the same reagent lot.

\section{Markers of lipid profile/muscle workload/exercise-induced stress}

The hematological parameters that were considered in the current pilot study were measured in a Healthcare structure Synlab ${ }^{\oplus}$ Pavia (Italy/EU). The parameters were the following: markers of lipid profile (Triglycerides, Total Cholesterol, HDL), markers of muscle workload (Creatinine, CPK, LDH, HGH, IGF-1) and markers of exerciseinduced stress i.e. steroid hormones (Testosterone and Cortisol). Creatinine, a waste product from the normal breakdown of muscle tissue, was used as a test of kidney function [25]. Both $\mathrm{CPK}$ and $\mathrm{LDH}$, which have important roles in cell energy processes, were used to assess damage to muscle tissues induced by injury [26]. We also assessed the blood concentrations of the polypeptide hormone $\mathrm{HGH}$ that enhances muscle mass, promotes lipolysis and gluconeogenesis, and stimulates the synthesis of IGF-1 in the liver. In addition to HGH, we measured IGF-1 because its synthesis can be altered by different factors, such as undernutrition, physical exercise status, stress levels, growth hormone insensitivity and lack of growth hormone receptors. IGF-1 enhances bone density, lean muscle mass, weight loss, carbohydrate metabolism and encourages cell regeneration and tissue repair [27].

\section{Markers of oxidative status and antioxidant parameters}

Given that the best approach in assessing oxidative stress seems to be the evaluation of the balance between antioxidants and by-products of oxidative reactions in the organism [28], we decided to investigate markers of oxidative damage (Malondialdeyde (MDA), Protein Carbonyl content (PCc) and 8-hydroxy-2' -deoxyguanosine (8-OHdG) [29]) and markers of antioxidant ability (the enzyme activities of Glutathione peroxidase (GPx), Speroxide dismutase (SOD) and Catalase (CAT)). We also used the DPPH test to determine the free radical scavenging activity, by evaluating the reagent 1,l'-diphenyl-2-picrylhydrazyl. The measurements were carried out in triplicate in the same laboratory. The mean of the three measurements was calculated and adopted. Their concentration and activities were assessed measuring absorbance by an UV-VIS spectrophotometer (Shimadzu UV1800) and a microplate spectrophotometer (BioTek ELx800).

MDA is one of the most reactive lipoperoxides $\mathrm{ROOH}$ (LPO), produced during the lipid peroxidation cascade of the PUFAs of the biological membranes, which are particularly susceptible to ROS-mediated oxidation due to their high double $\mathrm{C}=\mathrm{C}$ bond content. The reactive and radical species has sufficient activity to steal a hydrogen atom from a lipid methyl group, inducing a lipid peroxidation cascade [30]. The main effect of lipid peroxidation on biological membranes is the overall decrease in their fluidity and severe damage to the membrane proteins [31]. Lipid peroxidation was investigated by quantifying MDA value in urine and plasma by the Erdelmeier method [32]. In this assay, two molecules of MDA react with $\mathrm{N}$-methyl2-phenilindole $10.3 \mathrm{mmol} / \mathrm{l}$, at $45^{\circ} \mathrm{C}$ and $\mathrm{pH} 3.6$ for one hour to produce a stable complex that has maximum absorbance at $\lambda 586 \mathrm{~nm}$. The test was carried out in basal condition using the non-pretreated samples urine and plasma and in a stimulated condition using a pretreated sample of plasma with an oxidative agent, such as $\mathrm{CuSO}_{4}$ $0.5 \mathrm{mmol} / \mathrm{l}$, at $37^{\circ} \mathrm{C}$ for one hour.

PCc derived from protein carbonylation, an oxidation promoted by reactive oxygen species. It usually refers to a process that forms reactive ketones or aldehydes that 
are capable of reacting with the reagent 2,4-dinitrophenylhydrazine (DNPH) to form hydrazones. Protein oxidative damage involves both the loss of thiol groups and modifications to amino acids that constitute the polypeptide chain, in particular histidine. PCc is used as a marker of oxidative damage, which acts primarily on the side chains of the aminoacyl residues lysine, arginine, proline and threonine [33]. PCc was measured in RBC according to Levine and colleagues [34] after reaction with 2,4-Dinitrophenylhydrazine (DNPH) $10 \mathrm{mmol} / \mathrm{l}$ at $25^{\circ} \mathrm{C}$ for one hour. Streptomycine sulfate $1 \%$ was used to decrease nucleic acid contamination. At the end of the reaction, the carbonyl content was calculated from the maximum absorbance $(\lambda 360-390 \mathrm{~nm})$ and expressed as nmol on the amount of total proteins (mg), obtained applying the Lowry method [35].

ROS damage DNA either by an indirect mechanism or by direct interaction with molecules, forming 8-hydroxy2 '-deoxyguanosine (8-OHdG), a stable product derived from the attack of hydroxyl radical on guanine residues in the presence of oxygen or other oxidizing agents [36] and considered as a good biomarker of oxidative DNA damage [37]. The concentration in the urine was measured using the High Sensitivity-DNA/RNA oxidative Damage Elisa kit (Item № 589,320 Cayman Chemical, Michigan 48,108 USA) according to the manufacturer's instructions (normal value: $10-3000 \mathrm{pg} / \mathrm{ml}$ ). This immunoassay for the measure of DNA/RNA oxidative damage detects at $\lambda 405-420 \mathrm{~nm}$ all three oxidized guanine species: 8-hydroxy-2'-deoxyguanosine from DNA, 8hydroxyguanosine from RNA, and 8-hydroxyguanine from either DNA or RNA.

The endogenous antioxidant capacity was evaluated by measuring antioxidant parameters such as the enzyme activities in blood (RBC) and the total free radical scavenging activity in saliva, applying colorimetric assays. GPx activity was determined following the formation of NADP+ according to the method of Flohe \& Gunzler (1984) [38]. SOD activity was evaluated according to the capability of the samples to inhibit the reduction of cytochrome $\mathrm{C}$ with xanthine/xanthine oxidase, using the method developed by Flohe \& Otting (1984) [39]. CAT activity was determined by measuring the decomposition of $\mathrm{H}_{2} \mathrm{O}_{2}$ into $\mathrm{H}_{2} \mathrm{O}$, at $25^{\circ} \mathrm{C}$ and $\mathrm{pH} 7.0$, according to the method of Aebi (1984) [40]. The total free radical scavenging activity of low-molecular-weight nonenzymatic fraction (LMNEF) of whole saliva was determined by using the reagent 1,l'-diphenyl-2-picrylhydrazyl (DPPH), using the method developed by Atsumi (1999) [41]. The DPPH is a relatively stable compound in alcoholic solution with a peak absorbance at $\lambda 517 \mathrm{~nm}$. The radical scavenging activity of the whole saliva was determined in terms of the decreasing rate of absorbance detected at $517 \mathrm{~nm}$ in a $40 \%$ ethanol-DPPH solution ( $\mathrm{pH}$ 7.4) at room temperature.
The free radical scavenging activity was calculated as [(A517 control - A517 sample) / A517 control], representing the concentration of DPPH scavenged for $1 \mathrm{ml}$ of saliva.

\section{Markers of exercise-induced stress: steroid hormones in saliva}

Testosterone is a steroid hormone secreted from the Leydig cells of the testes under hypothalamic and pituitary control defining the hypothalamo-pituitary-testicular (HPT) axis. It has both anabolic and anti-catabolic effects on muscle tissue [42]. Cortisol is a steroid hormone released from the adrenal cortex stimulated by an Adreno Corticotropic Hormone (ACTH); it is involved in the response to stress [43]. The ratio between the concentration of testosterone and cortisol $(\mathrm{T} / \mathrm{C})$ is frequently used as an indication of the level of exercise-induced stress. Alterations in the concentration of these hormones are responsible for modulating several responses induced by training, such as hypertrophy and strength gain. Regarding this aspect, the balance between these anabolic/catabolic hormones is often used as an overreaching index and as a predictive index of OTS [44]. In the current study, we measured these two hormones not only in the blood, but also in saliva. The latter was a non-invasive, stress-free alternative to serum, used for example to evaluate unbound steroids, such as testosterone and cortisol that show a correlation with their free form serum concentrations [45, 46]. The concentration of testosterone and cortisol in saliva was determined by a colorimetric competitive enzymelinked immunosorbent assay (ELISA) method (Diametra Diagnostic S.r.l., Spello - Perugia, Italy/EU): testosterone saliva ELISA (Item № DKO021); cortisol saliva ELISA (Item № DKO020). The antigen in the sample competes with the antigenic testosterone or cortisol, conjugated with horseradish peroxidase (HRP) for binding to the limited number of antibodies anti testosterone or cortisol, coated on a 96-well plate. The colorimetric enzyme-linked immunosorbent reaction was detected at $\lambda 450 \mathrm{~nm}$ by a microplate spectrophotometer (BioTek ELx800).

\section{Marker of inflammation}

TNF- $\alpha$ is a $17 \mathrm{kDa}$ polypeptide early mediator in the acute phase response of the inflammation process, with an important role in the initiation of the inflammatory cascade, including the induction of liver-produced acute phase proteins such as CRP, the activation and differentiation of monocytes and macrophages, the expression of major histocompatibility complex (MHC) class I and II, and the expression of adhesion molecules on endothelial cells [47]. The concentration of TNF- $\alpha$ in plasma was evaluated by an enzyme-linked immunosorbent assay (ELISA) method (Item № 589,201 Cayman Chemical, Michigan 48,108 USA), which gives TNF- $\alpha$ measurements within the range 
of $0-250 \mathrm{pg} / \mathrm{ml}$, typically with a limit of detection of $1 \mathrm{pg} /$ $\mathrm{ml}$. The colorimetric enzyme-linked immunosorbent reaction was detected at $\lambda 405-420 \mathrm{~nm}$ by a microplate spectrophotometer (BioTek ELx800).

\section{Statistical analysis}

Continuous variables were reported as mean values \pm standard deviation (SD), and were analyzed using nonparametric tests (i.e., Friedman and Wilcoxon test, as appropriate), since these variables were not normally distributed (based on the Shapiro-Wilk statistic). All tests were two-sided and a $p$-value of less than 0.05 was considered as statistically significant. Since multiple measures were obtained from the same individuals, we used a Principal Component Analysis (PCA) to explore the relationships among the physiological parameters. The first 5 principal components $(\mathrm{PC}$, all with eigenvalue $>1$ ) which accounted for $60 \%$ of total variance and their scores were used as a set of independent variables in the subsequent analysis. In order to observe the consequences of $\mathrm{n}-3$ supplementation in Athletes and Sedentary subjects, we followed the procedure described by Adams and Collyer (2009) [48]: firstly, we computed the vector of physiological change (size) as the difference among the five PC scores before and after supplementation; then, we estimated the difference in direction of physiological change of Athletes and Sedentary subjects as the angle among their vectors of physiological change. The significant differences in size and angle were calculated by two PERMANOVA (Permutational Multivariate Analysis of Variance) with 9999 permutations. Statistical analyses were performed using R ver. 3.2.2 (R Development Core Team, 2015) [48, 49].

For each parameter, two Mixed Models were applied: one contained the status (Athlete or Sedentary), supplementation and their interaction as fixed effects, and the identity of the individual as a random effect; and the other was the same as the first, but without the interaction between status and supplementation. To evaluate whether the expression of the parameters in Athletes before and after supplementation was different from that of Sedentary subjects before and after supplementation, i.e. whether the interaction between the factors was significant, the two models were compared with a likelihood Ratio Test.

Linear correlation between parameters was measured using the Pearson correlation coefficient (with $R>0.5$ indicating a good correlation) and it was considered significant with values of $\mathrm{t}=6.16, \mathrm{df}=8, p$-value $<0.001$.

\section{Results}

The results of our study suggested that supplementation with n-3 PUFAs created a synergic variation in the parameters from a baseline state (T0) to a treated state after supplementation (T1), in terms of size and process, which was significantly different in Athletes compared to Sedentary subjects.

\section{Dietary analysis}

The data obtained using a 4-day food diary analysis of n3 and n-6 intake values for Athletes (Table 2) and Sedentary subjects (Table 3 ) were compared with one value (n-3: $1 \%$ of total energy intake; $n-6: 6 \%$ of total energy intake) chosen from the RI values defined by the Italian LARN [21]. The obtained ratio $n-6 / n-3$ for Athletes and Sedentary subjects was not different from the LARN value 6.00. However, both groups showed a lower intake of $n-3$ than the RI, even though the energy needs were sufficient.

\section{Analysis of relationships among parameters}

Data were analyzed with PCA to explore the relationships among the parameters. The first 5 principal components ( $\mathrm{PC}$, all with eigenvalue $>1$ ), accounting for $60 \%$ of total variance (Table 4), and their scores (Fig. 1) were used as a set of independent variables in the subsequent analysis. The principal components that seemed to explain most of the differences between Athletes and Sedentary subjects, before and after supplementation, were the 2 nd and the 5th components (Figs. 1 and 2).

\section{Variation in lipid profile and muscle workload}

Considering Triglycerides, total Cholesterol and Cholesterol HDL, the mean values were inside the normal range indicated by laboratory standard references (Triglycerides $20-150 \mathrm{mg} / \mathrm{dl}$; total cholesterol $<200 \mathrm{mg} / \mathrm{dl}$; Cholesterol $\mathrm{HDL}>40 \mathrm{mg} / \mathrm{dl}$ ) for both groups (Table 5). Concerning triglycerides values, only the Athlete group showed a significant reduction after 8 weeks of n-3 supplementation (Table 5), but if we considered how biological parameters affected each component indicated in Table 4, we observed that total Cholesterol decreased more markedly in Athletes compared to Sedentary subjects.

Regarding muscle workload, the Athletes showed a significant increase in creatinine after 8 weeks of supplementation (Table 6) (Laboratory standard references: male $0.70-1.30 \mathrm{mg} / \mathrm{dl}$; female $0.44-1.00 \mathrm{mg} / \mathrm{dl}$ ); this could be due to the protein-rich diet of the Athletes and to the physical exercise workload. On the contrary, CPK (standard laboratory reference indicates an optimal CPK value < $200 \mathrm{U} / \mathrm{l}$ in the blood) and LDH (standard laboratory reference indicates optimal LDH values: 125-243 U/l) results showed that there were no significant variations before or after supplementation in either group (Athletes or Sedentary subjects), as shown in Table 6, but the mean values of the Athletes were higher than those of the Sedentary subjects for both parameters (Table 6). Pearson correlation coefficient $(R=0.86)$ indicated a high correlation between CPK and LDH in blood (Table 7). 
Table 2 Dietary values for Athletes

\begin{tabular}{|c|c|c|c|c|c|c|c|}
\hline & Energy needs & N-3 tot (over 4 days) & LARN n-3 (1\%En) & $\mathrm{N}-6$ tot (over 4 days) & LARN n-6 (6\%En) & RATIO (n-6/n-3) & $\begin{array}{l}\text { LARN RATIO } \\
(\mathrm{n}-6 / \mathrm{n}-3)\end{array}$ \\
\hline & (Kcal) & (g) & (g) & (g) & (g) & $(n-6 / n-3)$ & $(n-6 / n-3)$ \\
\hline mean & 2355 & 1.58 & 2.62 & 9.23 & 15.70 & 6.11 & 6.00 \\
\hline SD & 505 & 1.02 & 0.56 & 6.93 & 3.37 & 1.89 & 0.00 \\
\hline \multicolumn{2}{|c|}{$p$ value $n-3$} & 0.001 & & & & & \\
\hline \multicolumn{2}{|c|}{$p$ value $n-6$} & & & 0.002 & & & \\
\hline \multicolumn{3}{|c|}{$p$ value ratio $n-6 / n-3$} & & & & 0.827 & \\
\hline$\Delta \mathbf{n}-\mathbf{3}$ & & $39.69 \%$ & & & & & \\
\hline \multicolumn{2}{|l|}{$\Delta \mathrm{n}-6$} & & & $45 \%$ & & & \\
\hline
\end{tabular}

The results showed that there were no significant differences among values before supplementation, and no variations in values after supplementation in either group (Athletes or Sedentary subjects) for both biomarkers HGH and IGF-1 (Table 8), which had optimal $\mathrm{HGH}$ standard laboratory reference values: male $<3.00$, female $<6.00 \mathrm{ng} / \mathrm{ml}$; IGF-1 values (16-24 years old): $150-480 \mathrm{ng} / \mathrm{ml}$.

\section{Variations in the markers of oxidative status and antioxidant parameters}

MDA. The $n-3$ supplementation induced a significant reduction in the lipid peroxidation marker (MDA) after 8 weeks of treatment in plasma for both Athletes and Sedentary subjects, whereas, the removed metabolite in urine showed a significant reduced value only in Athletes after supplementation and not in Sedentary subjects (Table 9). Moreover, MDA decreased more markedly in Athletes than in Sedentary subjects, showing that the supplementation had different effects depending on the status (Athletes or Sedentary subjects) (Fig. 3).
PCc. Even though proteins are easy targets of oxidative modifications as induced by ROS and lipid peroxidation products (MDA and HNE), oxidized proteins are harmful to the maintenance of cellular homeostasis as they require rapid removal by proteolytic digestion [33, 34]; indeed, our results, as reported in Table 9, did not show differences between groups and variation of the PCC values after 8 -weeks of supplementation, suggesting that neither physical exercise nor n-3 PUFA supplementation could affect protein carbonylation.

8-OHdG. This parameter of oxidative damage on DNA, such as PCc, did not show significant differences between values before and after treatment of $n-3$ supplementation for 8 weeks (Table 9).

The enzymatic antioxidant activities of GPx and CAT, which convert $\mathrm{H}_{2} \mathrm{O}_{2}$ to $\mathrm{H}_{2} \mathrm{O}$, increased after 8 weeks of supplementation in both groups: Athletes and Sedentary subjects (Table 10). However, the supplementation did not affect SOD activity, which promoted the dismutation of superoxide into $\mathrm{H}_{2} \mathrm{O}_{2}$ (Table 10). Regarding the antioxidant capacity evaluated as DPPH scavenging in saliva, the results, reported as the concentration of $\mathrm{DPPH}$

Table 3 Dietary values for Sedentary subjects

\begin{tabular}{|c|c|c|c|c|c|c|c|}
\hline & Energy needs & $\mathrm{N}-3$ tot (over 4 days) & LARN n-3 & $\mathrm{N}-6$ tot (over 4 days) & LARN n-6 & RATIO (n-6/n-3) & $\begin{array}{l}\text { LARN RATIO } \\
(n-6 / n-3)\end{array}$ \\
\hline & (kcal) & (g) & (g) & (g) & (g) & $(n-6 / n-3)$ & $(n-6 / n-3)$ \\
\hline mean & 2183 & 1.32 & 2.43 & 5.26 & 14.56 & 4.50 & 6.00 \\
\hline SD & 323 & 0.53 & 0.36 & 1.70 & 2.16 & 2.03 & 0.00 \\
\hline \multicolumn{2}{|c|}{$p$ value $n-3$} & 0.009 & & & & & \\
\hline \multicolumn{2}{|c|}{$p$ value $n-6$} & & & 0.001 & & & \\
\hline \multicolumn{2}{|c|}{$p$ value ratio $n-6 / n-3$} & & & & & 0.211 & \\
\hline \multicolumn{2}{|l|}{$\Delta \mathrm{n}-\mathbf{3}$} & $46.17 \%$ & & & & & \\
\hline \multicolumn{2}{|l|}{$\Delta n-6$} & & & $31.88 \%$ & & & \\
\hline
\end{tabular}


Table 4 Principal components

\begin{tabular}{llllll}
\hline & Comp-1 & Comp-2 & Comp-3 & Comp-4 & Comp-5 \\
\hline MDA-urine & -0.25 & $0.31^{*}$ & -0.04 & 0.02 & $0.51^{*}$ \\
DPPH-saliva & -0.19 & $0.43^{*}$ & -0.25 & 0.12 & 0.04 \\
Testosterone-blood plasma & -0.42 & -0.28 & 0.13 & 0.17 & 0.12 \\
Cortisol-blood plasma & 0.3 & -0.21 & -0.05 & 0.04 & $0.46^{*}$ \\
TNF- $\alpha$ - blood plasma & -0.22 & 0.3 & -0.38 & 0.32 & -0.12 \\
Creatinine-blood & 0.1 & $-0.46^{*}$ & -0.19 & $0.36^{*}$ \\
Total cholesterol-blood & 0.17 & -0.11 & -0.63 & -0.15 & -0.12 \\
Cholesterol-HDL-blood & 0.2 & -0.02 & -0.39 & -0.65 & -0.16 \\
Tryglicerides-blood & -0.01 & -0.13 & -0.3 & 0.21 & -0.08 \\
CPK & -0.46 & -0.16 & 0.03 & -0.13 & -0.29 \\
LDH & -0.44 & -0.03 & -0.04 & 0.12 & $-0.33^{*}$ \\
HGH & 0.19 & $0.4^{*}$ & 0.25 & 0.18 &
\end{tabular}

Principal components ( $\mathrm{PC}$, all with eigenvalue $>1$ ), accounting for $60 \%$ of total variance, and their scores. The values indicate how the biological parameters affect each component. The symbol (*) indicates the relevant effect on the component

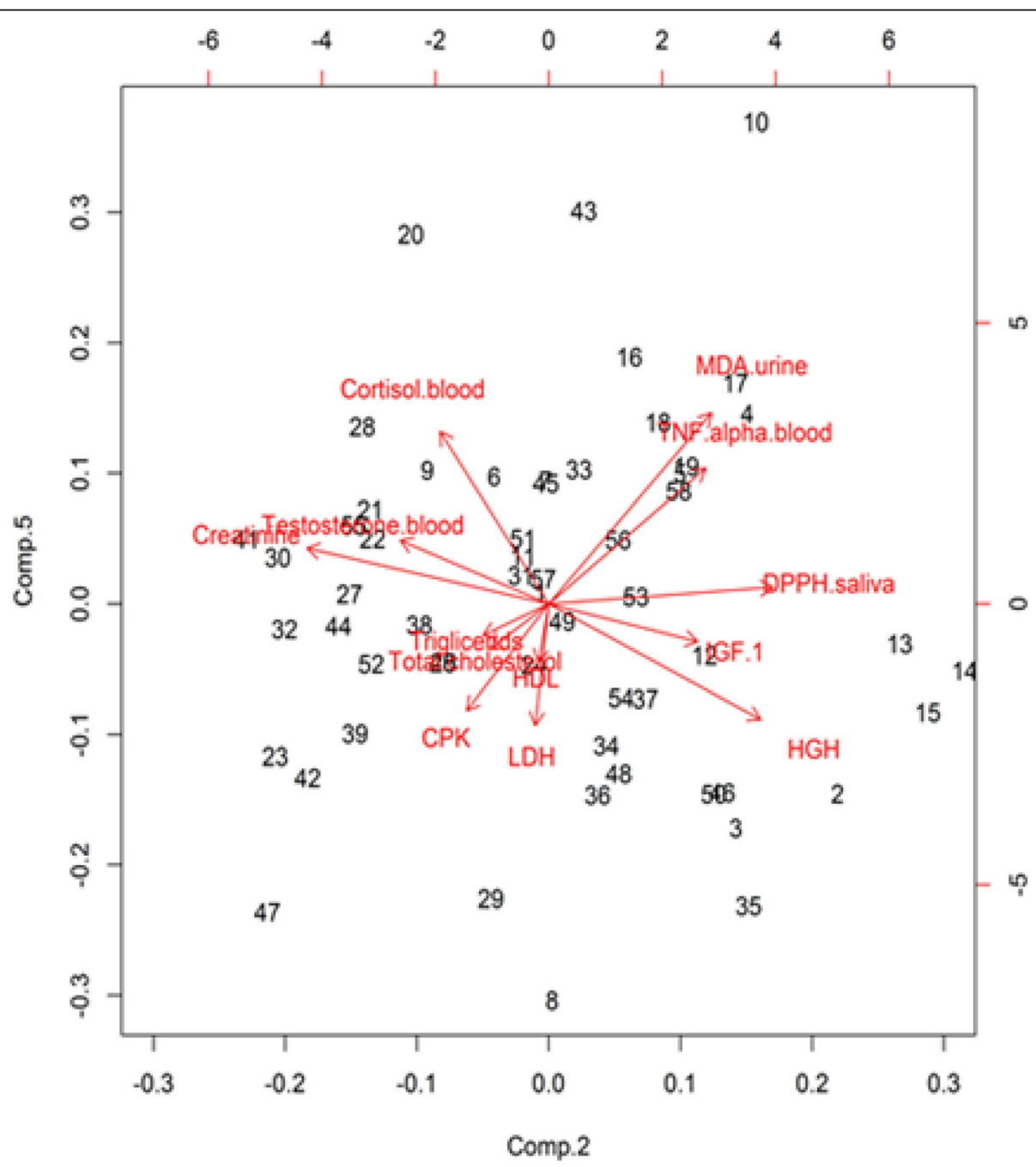

Fig. 1 PCA graph. Components graph showing the two selected components (Comp 2 and Comp 5) and parameters 


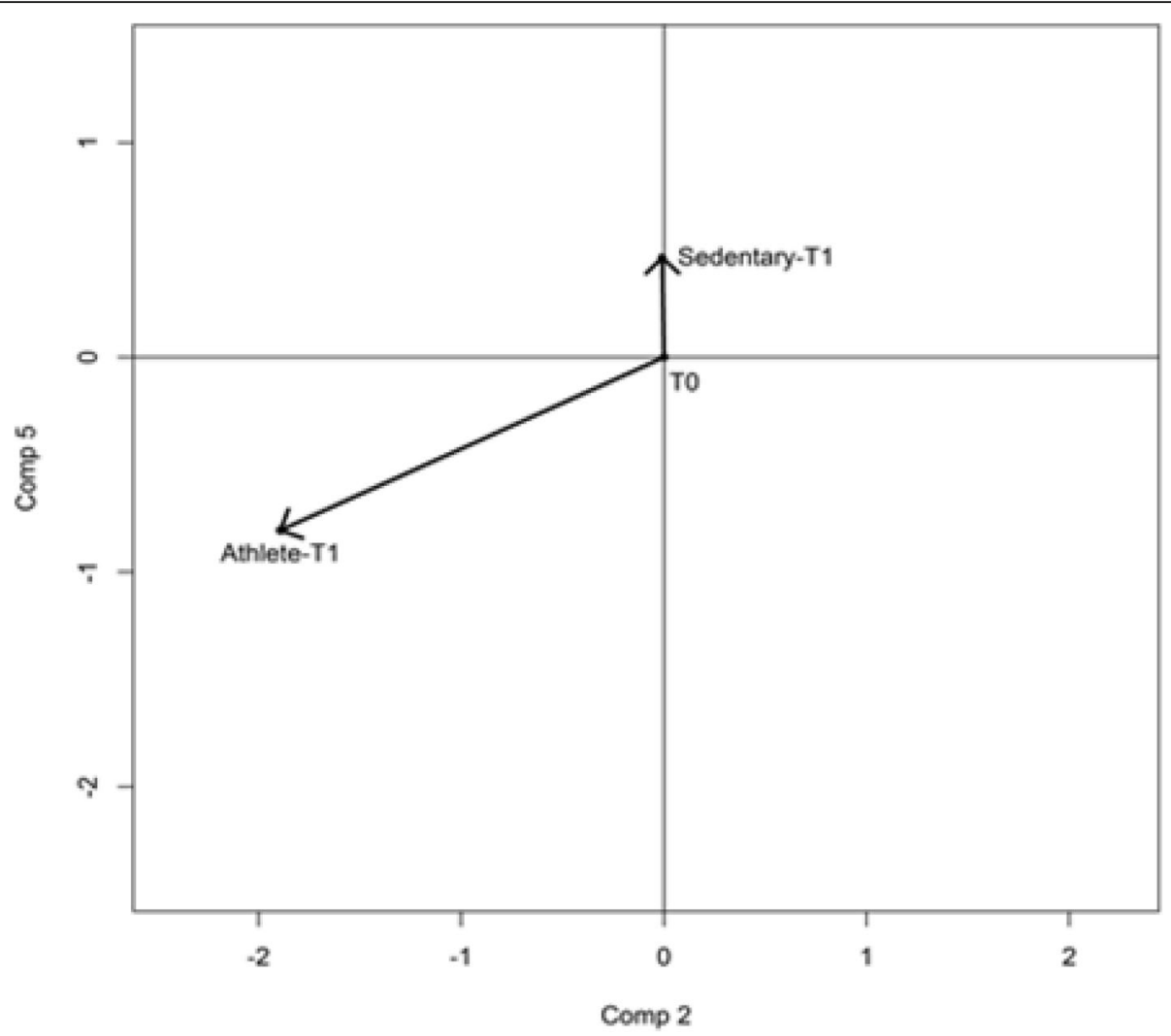

Fig. 2 Multivariate Analysis of Variance. Difference in direction of physiological change of Athletes and Sedentary subjects as the angle between their vectors of physiological change considering Components (Comp) 2 and 5

( $\mu \mathrm{mol}$ ) scavenged by $1 \mathrm{ml}$ of saliva, showed a decrease in the total free radical scavenging activity of LMNEF of whole saliva. The DPPH value decreased more in Athletes than in Sedentary subjects (Table 10, Fig. 4), suggesting that the supplementation had a negative effect on the scavenging activity of saliva and that the effects were different in the two groups (Athletes or Sedentary subjects) (Fig. 4).

\section{Variation in exercise-induced stress markers}

There was a multivariate significant difference between Athletes and Sedentary subjects: Cortisol tended to

Table 5 Lipid profile

\begin{tabular}{|c|c|c|c|c|c|c|c|c|}
\hline & & T0 & SD & T1 & SD & $p$ value & $\Delta \%$ & \\
\hline \multirow[t]{4}{*}{ Triglycerides (mg/dl) } & ATHL & 82 & 34 & 64 & 22 & $2 * 10^{-4}$ & 21.95 & T1vsT0 \\
\hline & SED & 67 & 15 & 65 & 17 & 0.35 & 2.98 & T1vsT0 \\
\hline & & & & & & 0.26 & 18.29 & TO \\
\hline & & & & & & 0.90 & 1.54 & $\mathrm{T1}$ \\
\hline \multirow[t]{4}{*}{ Total Cholesterol (mg/dl) } & ATHL & 175 & 26 & 171 & 35 & 0.47 & 2.28 & T1vsT0 \\
\hline & SED & 180 & 18 & 184 & 21 & 0.41 & 2.17 & T1vsT0 \\
\hline & & & & & & 0.68 & 2.78 & TO \\
\hline & & & & & & 0.39 & 7.06 & $\mathrm{~T} 1$ \\
\hline \multirow[t]{4}{*}{$\mathrm{HDL}(\mathrm{mg} / \mathrm{dl})$} & ATHL & 50 & 9 & 50 & 11 & 1.00 & 0.00 & T1vsT0 \\
\hline & SED & 54 & 8 & 55 & 9 & 0.76 & 1.82 & T1vsT0 \\
\hline & & & & & & 0.21 & 8.09 & T0 \\
\hline & & & & & & 0.36 & 9.10 & T1 \\
\hline
\end{tabular}

Data are expressed as mean values \pm SD comparing two groups and two periods. Statistical analysis: repeated measures analysis of variance; level of significance: $\mathrm{p}<0.05 ; \Delta \%$ for the difference between mean values, T1vs T0 intra-group, Sedentary (SED) vs Athlete (ATHL) groups for T0 and T1 
Table 6 Muscle workload

\begin{tabular}{|c|c|c|c|c|c|c|c|c|}
\hline & & T0 & SD & T1 & SD & $p$ value & $\Delta \%$ & \\
\hline \multirow[t]{4}{*}{ Creatinine (mg/dl) } & ATHL & 0.730 & 0.068 & 0.850 & 0.077 & $8^{*} 10^{-4}$ & 14.12 & T1vsT0 \\
\hline & SED & 0.840 & 0.131 & 0.790 & 0.098 & 0.08 & 5.95 & T1vsT0 \\
\hline & & & & & & 0.23 & 13.09 & T0 \\
\hline & & & & & & 0.31 & 7.06 & $\mathrm{~T} 1$ \\
\hline \multirow[t]{4}{*}{ CPK (U/I) } & ATHL & 187 & 124 & 198 & 115 & 0.76 & 5.56 & T1vsT0 \\
\hline & SED & 56 & 21 & 73 & 39 & 0.11 & 23.29 & T1vsT0 \\
\hline & & & & & & 0.02 & 70.05 & TO \\
\hline & & & & & & 0.02 & 63.13 & $\mathrm{~T} 1$ \\
\hline \multirow[t]{4}{*}{ LDH (U/l) } & ATHL & 179 & 38 & 175 & 33 & 0.70 & 2.23 & T1vsT0 \\
\hline & SED & 152 & 50 & 139 & 30 & 0.30 & 8.55 & T1vsT0 \\
\hline & & & & & & 0.16 & 15.08 & T0 \\
\hline & & & & & & 0.02 & 20.57 & $\mathrm{~T} 1$ \\
\hline
\end{tabular}

Data are expressed as mean values \pm SD comparing two groups and two periods. Statistical analysis: repeated measures analysis of variance; level of significance: $p<0.05 ; \triangle \%$ for the difference between mean values, T1vs T0 intra-group, Sedentary (SED) vs Athlete (ATHL) groups for T0 and T1

decrease in Athletes, whereas it increased in Sedentary subjects after supplementation (Table 11). Pearson correlation coefficient $(R=0.91)$ indicated a high correlation for Testosterone in blood and saliva (Fig. 5). The same result was obtained for Cortisol, for which Pearson correlation coefficient $(R=0.90)$ indicated a high correlation in blood and saliva (Fig. 6).
Moreover, the correlation between parameters (Table 7) was evaluated, resulting in the following findings: 1) a strong correlation between the $\mathrm{T} / \mathrm{C}$ ratio in blood and the $\mathrm{T} / \mathrm{C}$ ratio in saliva with parameters from which they were generated; 2) a strong correlation between Testosterone in blood and in saliva (Fig. 5); 3) a strong correlation between Cortisol in blood and in saliva (Fig. 6).

Table 7 Analysis of Correlation

\begin{tabular}{|c|c|c|c|c|c|c|c|c|c|c|c|c|c|c|c|c|c|}
\hline & $\begin{array}{l}\text { MDA } \\
\text { urn }\end{array}$ & $\begin{array}{l}\text { DPPH } \\
\text { slv }\end{array}$ & $\begin{array}{l}\mathrm{T} \\
\text { slv }\end{array}$ & $\begin{array}{l}\text { C } \\
\text { slv }\end{array}$ & $\begin{array}{l}\mathrm{T} / \mathrm{C} \\
\text { slv }\end{array}$ & $\begin{array}{l}\text { T } \\
\text { bld }\end{array}$ & $\begin{array}{l}\text { C } \\
\text { bld }\end{array}$ & $\begin{array}{l}\text { T/C } \\
\text { bld }\end{array}$ & $\begin{array}{l}\text { TNFa } \\
\text { bld }\end{array}$ & Creat & $\begin{array}{l}\text { Cholest } \\
\text { TOT }\end{array}$ & $\begin{array}{l}\text { Cholest } \\
\text { HDL }\end{array}$ & Trigly & CPK & LDH & $\mathrm{HGH}$ & IGF-1 \\
\hline MDAurn & 1.00 & 0.32 & -0.12 & 0.75 & -0.44 & -0.06 & 0.56 & -0.04 & -0.12 & -0.37 & 0.48 & -0.26 & 0.82 & -0.01 & 0.28 & 0.12 & -0.39 \\
\hline DPPHslv & 0.32 & 1.00 & 0.04 & -0.02 & 0.02 & 0.09 & -0.26 & 0.29 & 0.13 & -0.14 & -0.37 & -0.23 & 0.42 & 0.38 & 0.57 & 0.04 & -0.28 \\
\hline T_slv & -0.12 & 0.04 & 1.00 & -0.20 & 0.67 & 0.91 & -0.04 & 0.73 & -0.40 & 0.48 & -0.04 & 0.02 & -0.18 & 0.59 & 0.56 & -0.80 & -0.19 \\
\hline C_slv & 0.75 & -0.02 & -0.20 & 1.00 & -0.77 & -0.26 & 0.90 & -0.48 & -0.19 & -0.48 & 0.51 & -0.07 & 0.45 & -0.53 & -0.26 & 0.13 & 0.08 \\
\hline T/C slv & -0.44 & 0.02 & 0.67 & -0.77 & 1.00 & 0.72 & -0.63 & 0.81 & -0.17 & 0.52 & -0.30 & -0.08 & -0.30 & 0.85 & 0.58 & -0.42 & -0.30 \\
\hline T_bld & -0.06 & 0.09 & $0.91^{*}$ & -0.26 & 0.72 & 1.00 & -0.16 & 0.88 & -0.47 & 0.43 & 0.04 & -0.11 & -0.10 & 0.74 & 0.71 & -0.76 & -0.49 \\
\hline C_bld & 0.56 & -0.26 & -0.04 & 0.90 & -0.63 & -0.16 & 1.00 & -0.51 & -0.05 & -0.13 & 0.71 & 0.17 & 0.17 & -0.56 & -0.42 & 0.00 & 0.25 \\
\hline T/C bld & -0.04 & 0.29 & 0.73 & -0.48 & 0.81 & 0.88 & -0.51 & 1.00 & -0.40 & 0.28 & -0.22 & -0.28 & 0.12 & 0.91 & 0.91 & -0.56 & -0.67 \\
\hline TNFabld & -0.12 & 0.13 & -0.40 & -0.19 & -0.17 & -0.47 & -0.05 & -0.40 & 1.00 & 0.37 & 0.09 & 0.14 & 0.03 & -0.29 & -0.36 & 0.30 & 0.18 \\
\hline Creat & -0.37 & -0.14 & 0.48 & -0.48 & 0.52 & 0.43 & -0.13 & 0.28 & 0.37 & 1.00 & 0.27 & 0.48 & -0.51 & 0.27 & 0.06 & -0.58 & 0.07 \\
\hline $\begin{array}{l}\text { Cholest } \\
\text { TOT }\end{array}$ & 0.48 & -0.37 & -0.04 & 0.51 & -0.30 & 0.04 & 0.71 & -0.22 & 0.09 & 0.27 & 1.00 & 0.39 & 0.10 & -0.27 & -0.21 & -0.04 & -0.05 \\
\hline $\begin{array}{l}\text { Cholest } \\
\mathrm{HDL}\end{array}$ & -0.26 & -0.23 & 0.02 & -0.07 & -0.08 & -0.11 & 0.17 & -0.28 & 0.14 & 0.48 & 0.39 & 1.00 & -0.57 & -0.33 & -0.36 & -0.01 & 0.66 \\
\hline Trigly & 0.82 & 0.42 & -0.18 & 0.45 & -0.30 & -0.10 & 0.17 & 0.12 & 0.03 & -0.51 & 0.10 & -0.57 & 1.00 & 0.09 & 0.43 & 0.23 & -0.59 \\
\hline CPK & -0.01 & 0.38 & 0.59 & -0.53 & 0.85 & 0.74 & -0.56 & 0.91 & -0.29 & 0.27 & -0.27 & -0.33 & 0.09 & 1.00 & 0.86 & -0.38 & -0.60 \\
\hline $\mathrm{LDH}$ & 0.28 & 0.57 & 0.56 & -0.26 & 0.58 & 0.71 & -0.42 & 0.91 & -0.36 & 0.06 & -0.21 & -0.36 & 0.43 & 0.86 & 1.00 & -0.39 & -0.73 \\
\hline $\mathrm{HGH}$ & 0.12 & 0.04 & -0.80 & 0.13 & -0.42 & -0.76 & 0.00 & -0.56 & 0.30 & -0.58 & -0.04 & -0.01 & 0.23 & -0.38 & -0.39 & 1.00 & 0.26 \\
\hline $\mid G F-1$ & -0.39 & -0.28 & -0.19 & 0.08 & -0.30 & -0.49 & 0.25 & -0.67 & 0.18 & 0.07 & -0.05 & 0.66 & -0.59 & -0.60 & -0.73 & 0.26 & 1.00 \\
\hline
\end{tabular}

The high values $(\mathrm{R}>0.5)$ indicate a significant correlation between the analyzed parameters. Abbreviations: bld blood; C Cortisol; Cholest Cholesterol; Creat Creatinine; s/v saliva; $T$ Testosterone; T/C RATIO T/C; Trigly Triglyceride; urn urine 
Table 8 Muscle Workload

\begin{tabular}{llllllll}
\hline & & T0 & SD & T1 & SD & $\boldsymbol{p}$ value & $\Delta \%$ \\
\hline HGH (ng/ml) & ATHL & 2.9 & 4.5 & 2.6 & 3.6 & 0.86 & 10.34 T1vsT0 \\
& SED & 4.5 & 5.8 & 5.6 & 4.4 & 0.54 & 19.64 T1vsT0 \\
& & & & & & 0.46 & 35.56 T0 \\
& & & & & & 0.09 & 53.57 T1 \\
IGF-1 (ng/ml) & ATHL & 290 & 142 & 306 & 115 & 0.68 & 5.23 T1 vsT0 \\
& SED & 271 & 78 & 274 & 81 & 0.82 & 1.09 T1 vsT0 \\
& & & & & & 0.76 & 6.55 T0 \\
& & & & & & 0.65 & 10.46 T1 \\
\hline
\end{tabular}

Data are expressed as mean values \pm SD comparing two groups and two periods. Statistical analysis: repeated measures analysis of variance; level of significance: $p<0.05 ; \triangle \%$ for the difference between mean values, T1vs T0 intra-group, Sedentary (SED) vs Athlete (ATHL) groups for T0 and T1

To maximize the diversity of parameters for statistical analysis, Testosterone and Cortisol measured in saliva and the two reports "ratio $\mathrm{T} / \mathrm{C}$ " were eliminated. Moreover, the $\mathrm{T} / \mathrm{C}$ ratio values in plasma blood and saliva for both groups was reported in Fig. 7. The T/C ratio tended to decrease after supplementation in both groups because Cortisol increased, but the variation was more evident in Sedentary subjects than in Athletes.
Variation in the marker of inflammation

Regarding the results of the inflammatory marker, the plasma concentration of TNF- $\alpha$ decreased more evidently in Athletes compared to Sedentary subjects (Table 12). The supplementation had different effects depending on the status (Athletes or Sedentary subjects), as reported in Fig. 8.

\section{Discussion}

The main difference between the two examined groups (Athletes and Sedentary subjects) before supplementation with about $4 \mathrm{~g} /$ day of $\mathrm{n}-3$ PUFAs, rich in EPA and DHA, for 8 weeks, was their physical exercise status. The results of the current study suggested that the n-3 supplementation created a synergic variation in the parameters from a baseline state (T0) to a treated state after supplementation (T1), in terms of size and modality, which was significantly different in Athletes compared to Sedentary subjects.

It is well known that intense and prolonged exercise is characterized by a large formation of radical oxygen and nitrogen compounds, as well as by an imbalance of homeostasis between pro-oxidant/anti-oxidant species and a general increase in the inflammatory state of the human body [2-5]. In this pilot study, the analysis of the results, obtained after n-3 fatty acid supplementation of

Table 9 Oxidative status: MDA, PCc, 8-OHdG

\begin{tabular}{|c|c|c|c|c|c|c|c|c|}
\hline & & T0 & SD & T1 & SD & $p$ value & $\Delta \%$ & \\
\hline \multirow[t]{4}{*}{ MDA URINE $(\mu \mathrm{mol} / \mathrm{ml})$} & ATHL & 0.030 & 0.017 & 0.019 & 0.011 & 0.02 & 36.67 & T1vsT0 \\
\hline & SED & 0.016 & 0.02 & 0.017 & 0.011 & 0.45 & 5.88 & T1vsT0 \\
\hline & & & & & & 0.10 & 46.67 & T0 \\
\hline & & & & & & 0.70 & 10.53 & T1 \\
\hline \multirow[t]{4}{*}{ MDA BASAL PLASMA $(\mu \mathrm{mol} / \mathrm{ml})$} & ATHL & 0.026 & 0.015 & 0.002 & 0.002 & $1 * 10^{-6}$ & 90.59 & T1vsT0 \\
\hline & SED & 0.003 & 0.002 & 0.001 & 0.001 & 0.01 & 58.82 & T1vsT0 \\
\hline & & & & & & $6^{*} 10^{-4}$ & 86.67 & T0 \\
\hline & & & & & & 0.30 & 41.67 & T1 \\
\hline \multirow[t]{4}{*}{ MDA STIMULATED PLASMA $(\mu \mathrm{mol} / \mathrm{ml})$} & ATHL & 0.053 & 0.017 & 0.016 & 0.003 & $7.0^{*} 10^{-8}$ & 69.19 & T1vsT0 \\
\hline & SED & 0.018 & 0.002 & 0.015 & 0.002 & $1 * 10^{-3}$ & 18.03 & T1vsT0 \\
\hline & & & & & & $2.7^{*} 10^{-5}$ & 65.41 & T0 \\
\hline & & & & & & 0.30 & 7.98 & T1 \\
\hline \multirow[t]{4}{*}{ PCc (nmol/mg) } & ATHL & 3.83 & 2.85 & 3.73 & 2.32 & 0.92 & 2.71 & T1vsT0 \\
\hline & SED & 3.50 & 2.12 & 5.31 & 1.61 & 0.1 & 34.01 & T1vsT0 \\
\hline & & & & & & 0.76 & 8.61 & T0 \\
\hline & & & & & & 0.09 & 29.75 & $\mathrm{~T} 1$ \\
\hline \multirow[t]{4}{*}{ 8-OHdG (ng/ml) } & ATHL & 27.87 & 22.50 & 56.47 & 45.60 & 0.11 & 50.65 & T1vsT0 \\
\hline & SED & 73.77 & 75.00 & 67.32 & 63.90 & 0.87 & 8.74 & T1vsT0 \\
\hline & & & & & & 0.2 & 62.36 & T0 \\
\hline & & & & & & 0.72 & 16.12 & $\mathrm{~T} 1$ \\
\hline
\end{tabular}

Data are expressed as mean values \pm SD comparing two groups and two periods. Statistical analysis: repeated measures analysis of variance; level of significance: $\mathrm{p}<0.05 ; \triangle \%$ for the difference between mean values, T1vs T0 intra-group, Sedentary (SED) vs Athlete (ATHL) groups for T0 and T1 


\section{MDA urine}

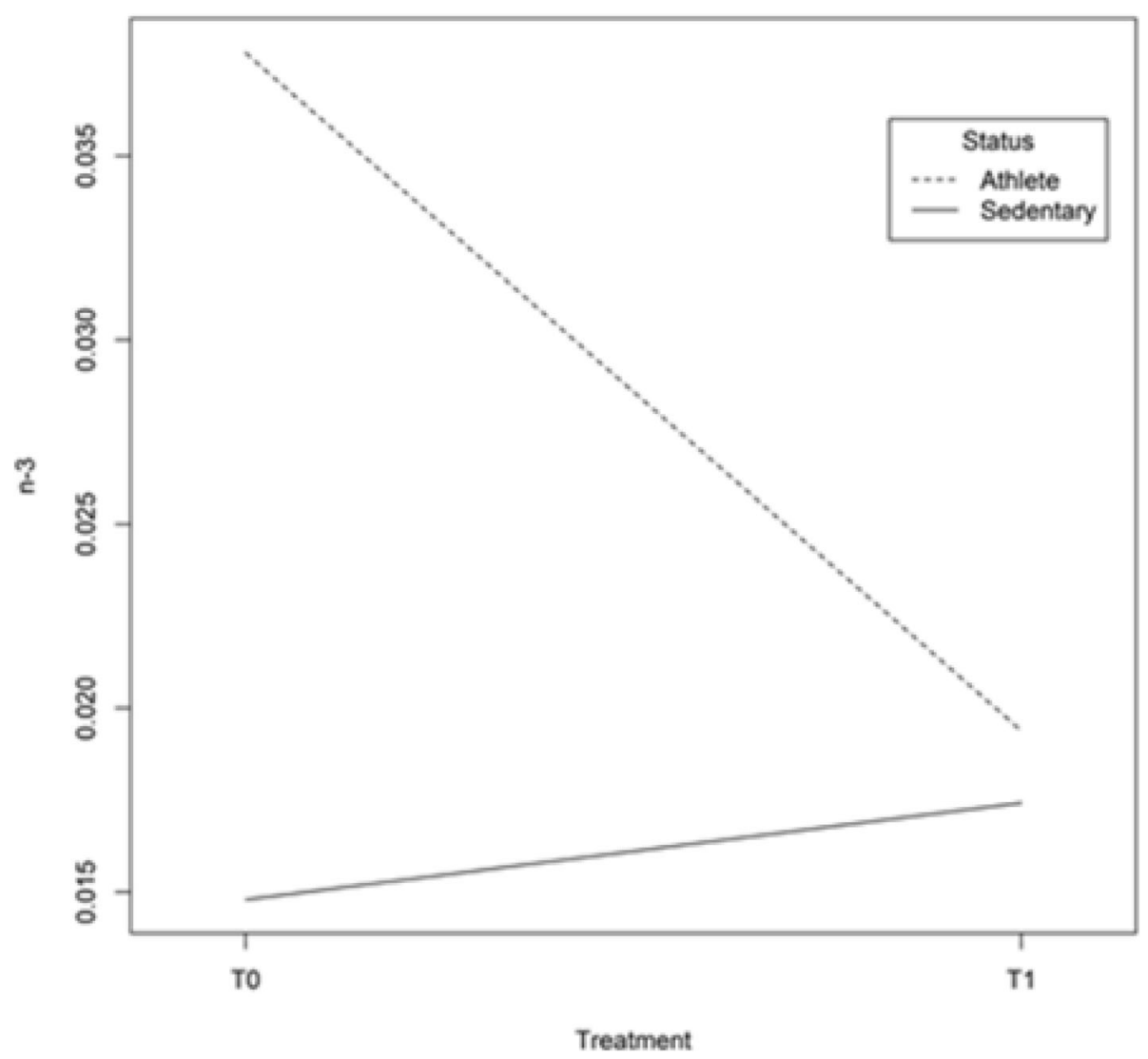

Fig. $3 \mathrm{MDA}$ in urine for Athletes and Sedentary subjects in different periods: before (T0) and after (T1) supplementation. Interactions between factors are cogent and significant $(\mathrm{L}$ ratio $=6.095124 ; p$ value $=0.0136)$

about $4 \mathrm{~g} /$ day for 8 weeks, highlighted some benefits in different areas. In particular, n-3 PUFA supplementation may affect oxidative stress, as suggested by the significant decrease in MDA, a marker of oxidative stress [28], and by the significant increase in enzyme antioxidant activities after supplementation. MDA decreased in Athletes more than in Sedentary subjects, suggesting that the treatment had different effects depending on the amount of exercise performed (Athletes or Sedentary subjects). On the contrary, the supplementation had no effect on the other markers of oxidative damage, such as carbonylated proteins and 8-OHdG. Therefore, these results lead us to believe that the localization of n-3, extracellular and trans membrane EPA and DHA, which act on phospholipids, may influence the activity of these fatty acids. Our study also showed promising results for enzymatic antioxidant capacity: supplementation was shown to improve the activity of GPx and CAT both in Athletes and Sedentary subjects.

Another important result was obtained regarding inflammation, namely that the consumption of about $4 \mathrm{~g} /$ day of n-3 PUFAs for 8 weeks was shown to reduce the concentration of pro-inflammatory cytokine TNF- $\alpha$, both in Athletes and Sedentary subjects, but TNF- $\alpha$ decreased more evidently in Athletes than in Sedentary subjects. This result leads us to hypothesize positive effects of n-3 supplementation on an inflammatory status, but the high variability of the data collected for Athletes (time T0) suggests the need to use a larger sample size than we used in this pilot study.

Moreover, the current investigation demonstrated that a linear correlation existed between the steroid hormones Testosterone and Cortisol measured in saliva and blood, according to literature data [50], validating the use of saliva samples as an innovative and non-invasive matrix instead of blood samples. The use of saliva samples was simple, quick, cheap, non-invasive and could be reproduced various times a day; these characteristics were very important considering a study involving Athletes because it was possible to take saliva samples during different competitions. As saliva samples are easily accessible, they can be used to assess competition or training conditions and for anti-doping purposes. The results of this pilot study showed that Cortisol 
Table 10 Antioxidant parameters: GPx, SOD, CAT, DPPH

\begin{tabular}{|c|c|c|c|c|c|c|c|c|}
\hline & & Tо & SD & $\mathrm{T} 1$ & SD & $p$ value & $\Delta \%$ & \\
\hline \multirow[t]{4}{*}{$\mathrm{GPx}(\mathrm{U} / \mathrm{gHb})$} & ATHL & 9.78 & 2.00 & 13.70 & 3.39 & $6.2 * 10^{-4}$ & 28.61 & T1vsT0 \\
\hline & SED & 6.55 & 2.97 & 17.99 & 4.55 & $4.8 * 10^{-4}$ & 63.61 & T1vsT0 \\
\hline & & & & & & $4^{*} 10^{-3}$ & 33.08 & T0 \\
\hline & & & & & & $1.7^{*} 10^{-3}$ & 23.83 & $\mathrm{~T} 1$ \\
\hline \multirow[t]{4}{*}{ SOD (U/gHb) } & ATHL & 722 & 180 & 761 & 129 & 0.24 & 5.05 & T1vsT0 \\
\hline & SED & 598 & 176 & 692 & 65 & 0.07 & 13.68 & T1vsT0 \\
\hline & & & & & & 0.13 & 17.26 & Tо \\
\hline & & & & & & 0.19 & 8.95 & $\mathrm{~T} 1$ \\
\hline \multirow[t]{4}{*}{ CAT $(\mathrm{mmol} / \mathrm{min})$} & ATHL & 0.007 & 0.002 & 0.010 & 0.006 & $3 * 10^{-3}$ & 38.68 & T1vsT0 \\
\hline & SED & 0.007 & 0.002 & 0.028 & 0.008 & $2^{*} 10^{-4}$ & 76.49 & T1vsT0 \\
\hline & & & & & & 0.76 & 2.98 & то \\
\hline & & & & & & $2 * 10^{-6}$ & 62.81 & $\mathrm{~T} 1$ \\
\hline \multirow[t]{4}{*}{ DPPH $(\mu \mathrm{mol} / \mathrm{ml})$} & ATHL & 0.589 & 0.102 & 0.242 & 0.173 & $1.4^{*} 10^{-7}$ & 58.91 & T1vsT0 \\
\hline & SED & 0.457 & 0.047 & 0.373 & 0.067 & 0.02 & 18.38 & T1vsT0 \\
\hline & & & & & & $3 * 10^{-3}$ & 22.41 & то \\
\hline & & & & & & 0.07 & 35.12 & $\mathrm{~T} 1$ \\
\hline
\end{tabular}

Data are expressed as mean values \pm SD comparing two groups and two periods. Statistical analysis: repeated measures analysis of variance; level of significance: $\mathrm{p}<0.05 ; \Delta \%$ for the difference between mean values, T1vs T0 intra-group, Sedentary (SED) vs Athlete (ATHL) groups for T0 and T1

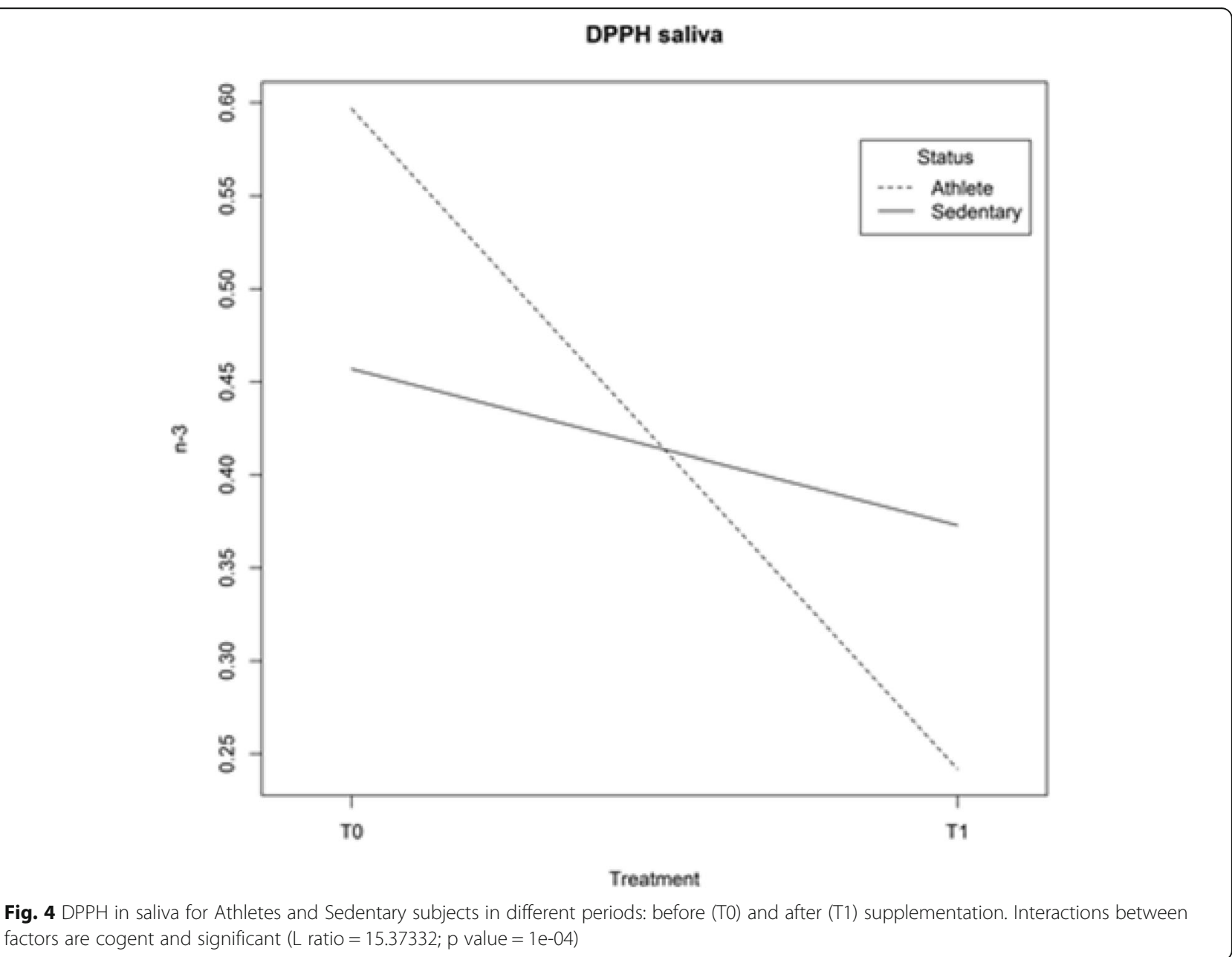


Table 11 Exercise-induced stress levels: Testosterone and Cortisol

\begin{tabular}{|c|c|c|c|c|c|c|c|c|}
\hline & & T0 & SD & T1 & SD & $p$ value & $\Delta \%$ & \\
\hline \multirow{4}{*}{$\begin{array}{l}\text { TOTAL TESTOSTERONE } \\
\text { Plasma }(\mathrm{ng} / \mathrm{ml})\end{array}$} & ATHL & 4.8 & 3.21 & 4.67 & 2.84 & 0.34 & 1.67 & T1vsT0 \\
\hline & SED & 2.53 & 3.59 & 2.19 & 2.72 & 0.26 & 13.44 & T1vsT0 \\
\hline & & & & & & 0.34 & 47.29 & T0 \\
\hline & & & & & & 0.26 & 53.10 & $\mathrm{~T} 1$ \\
\hline \multirow{4}{*}{$\begin{array}{l}\text { TESTOSTERONE } \\
\text { Saliva (pg/ml) }\end{array}$} & ATHL & 151 & 38 & 139 & 49 & 0.16 & 7.88 & T1vsT0 \\
\hline & SED & 108 & 57 & 113 & 41 & 0.41 & 4.17 & T1vsT0 \\
\hline & & & & & & 0.10 & 28.56 & T0 \\
\hline & & & & & & 0.28 & 19.07 & $\mathrm{~T} 1$ \\
\hline \multirow[t]{4}{*}{ CORTISOL Plasma (ng/ml) } & ATHL & 160 & 53 & 169 & 25 & 0.22 & 5.83 & T1vsT0 \\
\hline & SED & 176 & 35 & 216 & 44 & 0.01 & 18.43 & T1vsT0 \\
\hline & & & & & & 0.46 & 9.37 & T0 \\
\hline & & & & & & $3^{*} 10^{-3}$ & 21.50 & T1 \\
\hline \multirow[t]{4}{*}{ CORTISOL Saliva (ng/ml) } & ATHL & 11.11 & 5.12 & 8.54 & 1.06 & 0.15 & 23.13 & T1vsT0 \\
\hline & SED & 7.57 & 3.43 & 12.65 & 3.53 & 0.01 & 40.16 & T1vsT0 \\
\hline & & & & & & 0.28 & 31.86 & T0 \\
\hline & & & & & & 0.04 & 32.49 & T1 \\
\hline
\end{tabular}

Data are expressed as mean values \pm SD comparing two groups and two periods. Statistical analysis: repeated measures analysis of variance; level of significance: $p<0.05 ; \Delta \%$ for the difference between mean values, T1vs T0 intra-group, Sedentary (SED) vs Athlete (ATHL) groups for T0 and T1

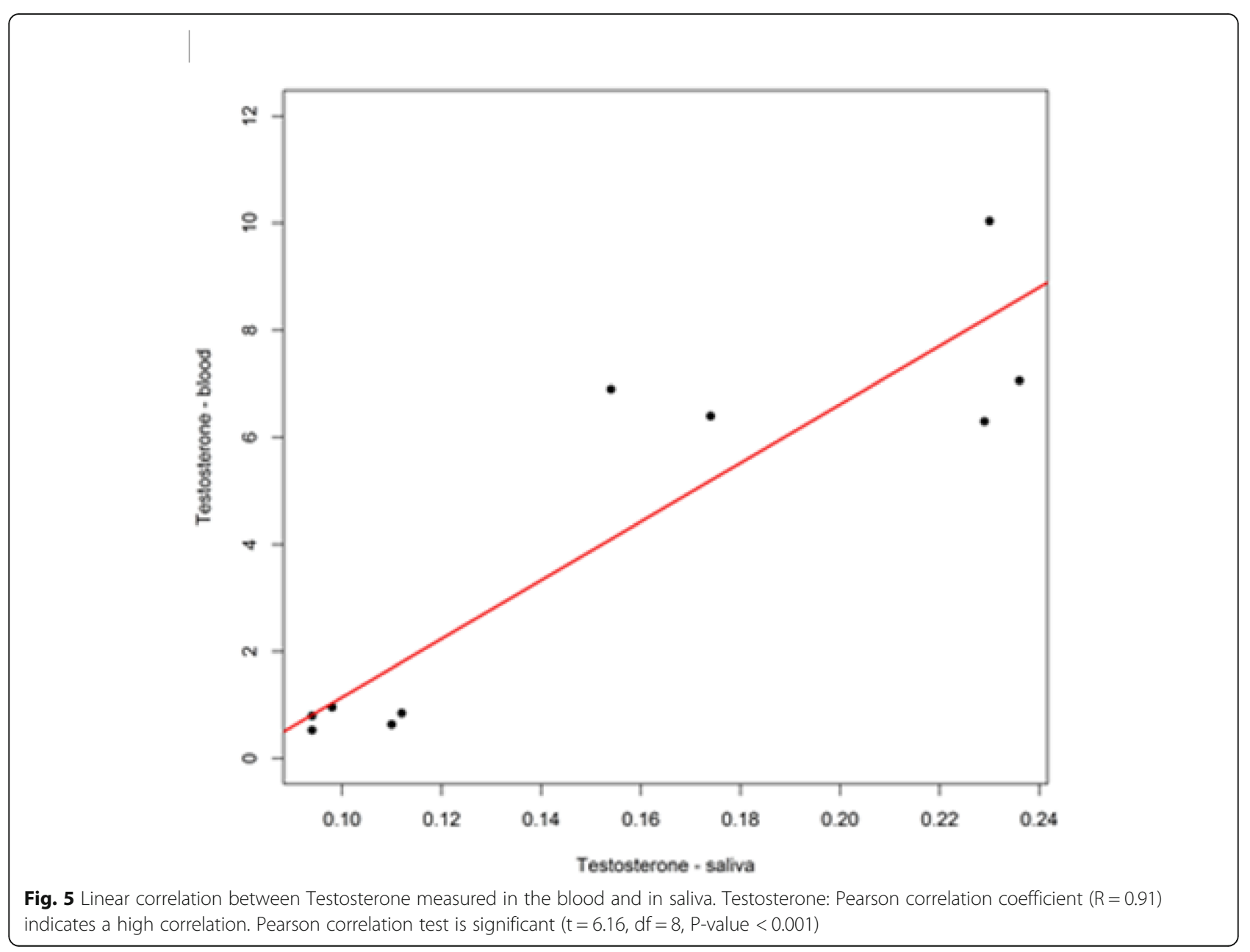




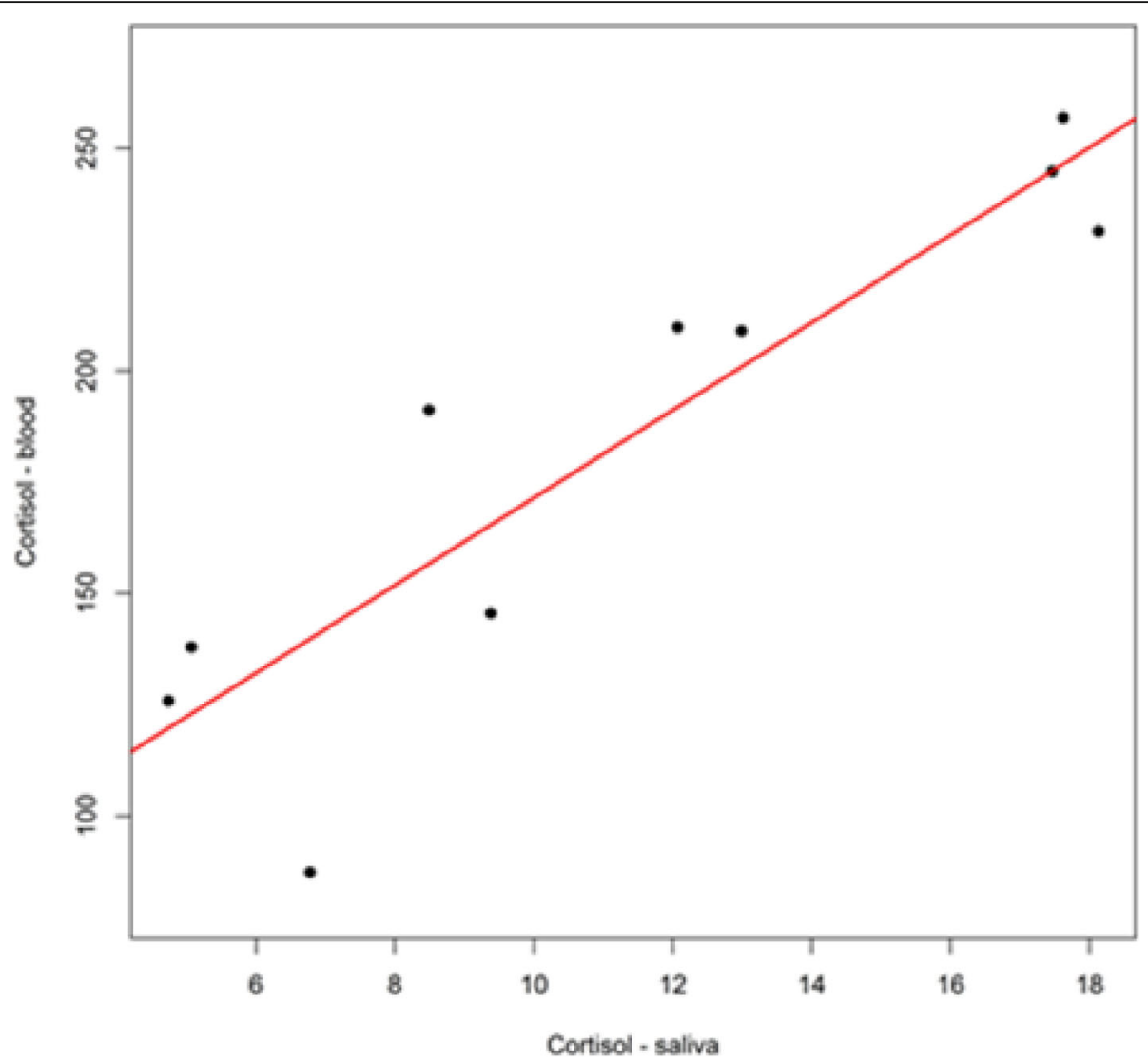

Fig. 6 Linear correlation between Cortisol measured in the blood and in saliva. Cortisol: Pearson correlation coefficient $(R=0.90)$ indicates a high correlation. Pearson correlation test is significant $(\mathrm{t}=5.83, \mathrm{df}=8, P$-value $<0.001)$

significantly increased in the Sedentary group after the supplementation period. There was a multivariate significant difference between Athletes and Sedentary subjects: Cortisol tended to decrease in Athletes, but increase in Sedentary subjects after supplementation. This hormone, known as the stress hormone, seemed to be more susceptible to change in Sedentary subjects than in Athletes. This observation could be due to the different lifestyle Sedentary subjects have compared to Athletes: the latter group have more stable hormone levels than the former group because
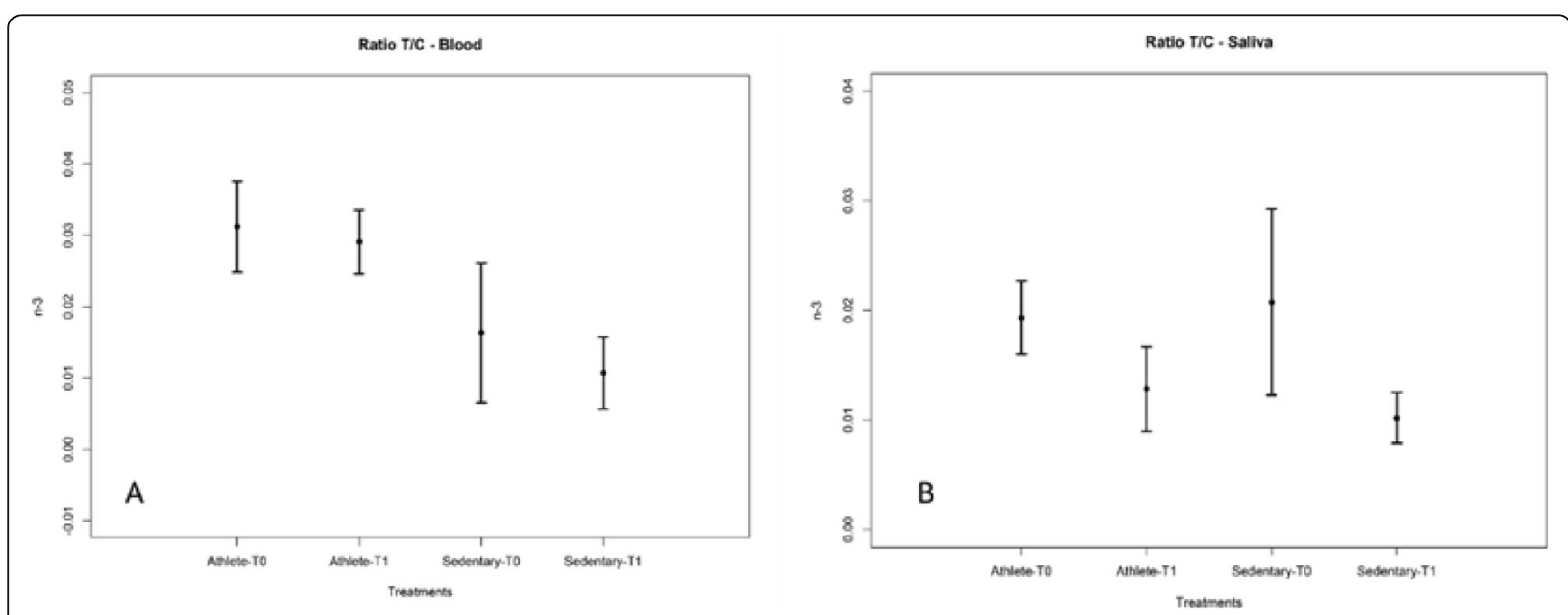

Fig. 7 T/C ratio in plasma blood (A) and saliva (B) for Athletes and Sedentary subjects in different periods: before (T0) and after (T1) supplementation. Data are expressed as mean \pm SD 
Table 12 Inflammatory marker: TNF-a

\begin{tabular}{lllllllll}
\hline & & T0 & SD & T1 & SD & $p$ value & $\Delta \%$ & \\
\hline TNF-a & ATHL & 54.57 & 64.80 & 0.05 & 18.80 & 0.02 & 99.91 & T1vsT0 \\
(pg/ml) & SED & 18.18 & 0.20 & 0.00 & 0.00 & 0.01 & 100.00 & T1vsT0 \\
& & & & & & 0.24 & 66.68 & T0 \\
& & & & & & 0.52 & 100.00 & T1 \\
\hline
\end{tabular}

Data are expressed as mean values \pm SD comparing two groups and two periods. Statistical analysis: repeated measures analysis of variance; level of significance: $p<0.05 ; \triangle \%$ for the difference between mean values, T1vs T0 intra-group, Sedentary (SED) vs Athlete (ATHL) groups for T0 and T1

of their daily workouts and more regular way of life. In this case, the increased Cortisol in Sedentary subjects could not be due to the $n-3$ supplementation because this increase was not found in Athletes either. It is likely that the effect of $n-3$ supplementation in Athletes is more evident because it is sustained by their ability to control their Cortisol level, which dramatically increases in agonist athletes, who are subjected to physical stress $[43,44]$. The ratio values did not indicate that subjects overreached, indeed all values were higher than 0.00035 and there was no decrease in ratio (>30\%), as reported by Adlercreutz and colleagues [51]. It is known that the hypothalamo-pituitary-adrenal (HPA) axis, which controls cortisol release, plays an important role in the adaptation to endurance training and acute response to exercise. Cortisol exerts catabolic effects on muscle tissue [43] and has important metabolic functions, such as influencing the metabolism of lipids, proteins and glucose. It increases the mobilization of fatty acids from fat reserves to active tissue and raises blood glucose [52]. Intense physical exercise increases Cortisol [53], which may inhibit protein synthesis with consequent increase in muscle mass by its catabolic effect [52].

According to the obtained dietary data, all subjects (Athletes and Sedentary subjects) showed a lower intake of n-3 PUFAs than the recommended intake by LARN 2014 [21]. After supplementation, the mobilization of fatty acids was further improved, as shown by the reduction in triglycerides and total cholesterol levels in the Athletes' blood. This effect was more evident in Athletes than in Sedentary subjects; on the contrary, there were no changes in HDL levels in either experimental group.

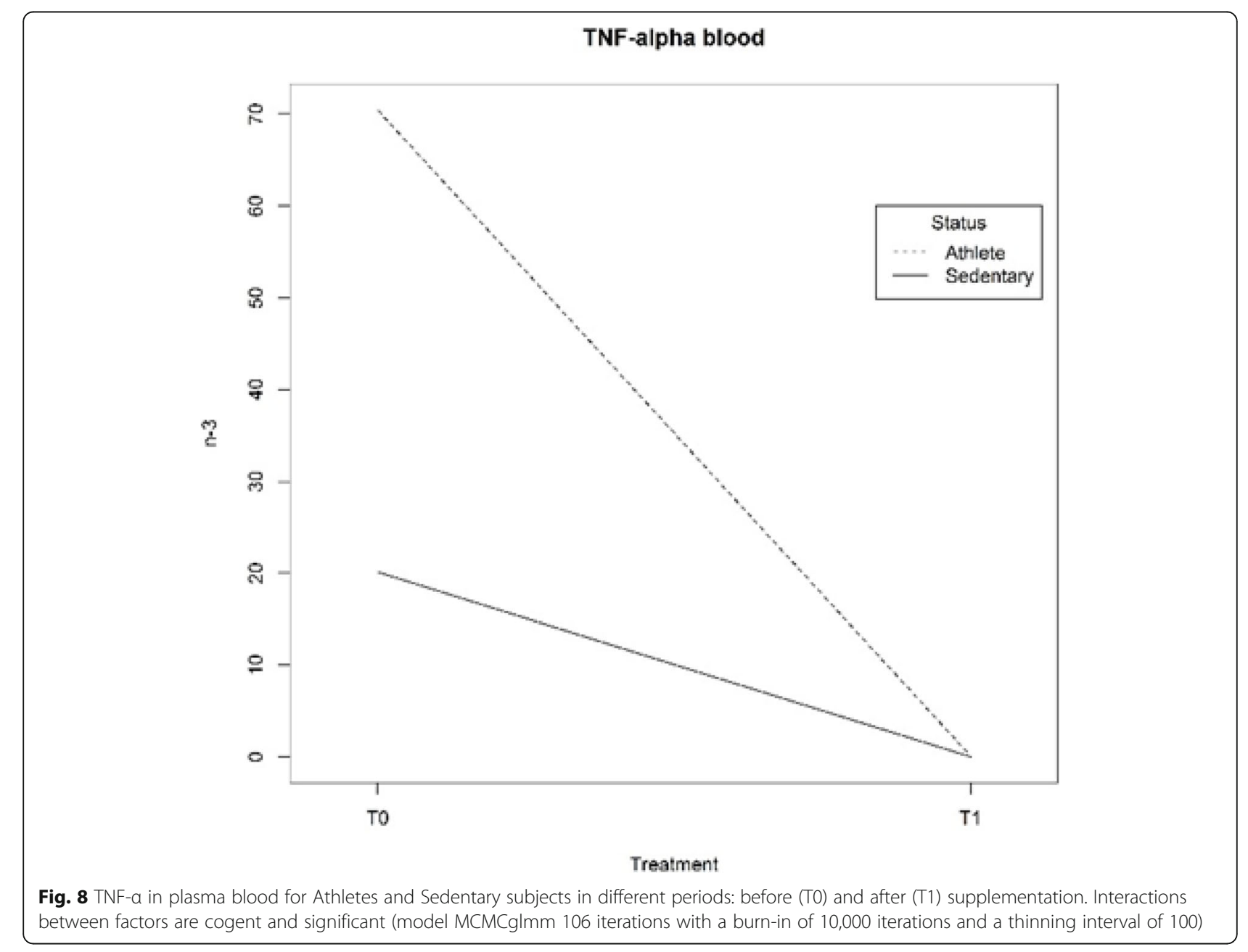


It is important to point out that all voluntary subjects were young people ( $<35$ years old), who showed basal lipid values that were within the normal standard range, so it would be interesting to plan a new study considering subjects with high cholesterol levels.

The Athlete group showed a significant increase in the creatinine value after 8 weeks of supplementation; this could be due to the Athletes' protein-rich diet. Moreover, the muscular work carried out by the Athlete group leads to high levels of LDH and CPK, as well as an accentuated oxidative status, particularly in Athletes, confirmed by the levels of MDA in plasma and urine that proved to be higher than reported in literature [54]. The study showed that there were no significant variations in muscle workload markers (CPK, LDH and growth hormones such as HGH and IGF-1) after n-3 PUFA supplementation in both experimental groups.

In summary, our pilot study suggested that strenuous physical exercise training leads to increased triglyceride levels, CPK, LDH, MDA, TNF- $\alpha$ and Gpx (not balanced by CAT increase), thus the endogenous antioxidant defenses are not sufficient to counteract oxidative stress (increased risk of overtraining syndrome).

Dietary supplementation with n-3 PUFAs (4 g/day for 8 weeks), enriched in EPA and DHA, decreased triglyceride levels, MDA and TNF- $\alpha$ and increased Gpx and CAT. Therefore, it may partially counteract oxidative stress, protecting middle- and long-distance running Athletes from the risk of overtraining.

Overall, our study showed a marked effect of $n-3$ supplementation in Athletes compared to Sedentary subjects; this effect may be due to an adaptation "hormesis" to stress conditions in athletes [55], expressed by a high threshold antioxidant capacity, contrasting oxidative status. Therefore, an interaction seemed to exist between two stimuli, physical exercise and $\mathrm{n}-3$ supplementation, on the evaluated markers.

For the future, it would be interesting to enlarge the inflammatory panel to include other inflammatory markers, such as interleukins (IL) IL-6 and IL-8. Moreover, it would be interesting to include a third experimental group of Athletes who practice regular but nonstrenuous physical exercise. Furthermore, considering that running-specific studies are scarce, this study increases the data available in scientific literature. Even though the data were obtained from a small sample, the results of the current study could highlight recommendations for future research to optimize running performance with nutritional interventions.

\section{Conclusions}

n-3 PUFA supplementation may be useful as a nutritional countermeasure to strenuous exercise-induced oxidative stress and inflammation in Athletes, but further in vivo studies in humans need to be carried out.

\section{Abbreviations}

AA: Arachidonic acid; ACTH: Adreno corticotropic hormone; Als: Adequate intakes; AP: Ascoli Piceno; ARs: Average requirements; ATHL: Athletes; bld: blood; BMI: Body mass index; C: Cortisol; CAT: Catalase;

Cholest: Cholesterol; Comp: Components; CPK: Creatine phosphokinase cps: capsule; creat: creatinine; slv: saliva; DHA: Docosahexaenoic acid; DNPH: 2,4-dinitrophenylhydrazine; DPPH: 1,1'-diphenyl-2-picrylhydrazyl; DRIv: Dietary Reference Intake values; EFSA: European Food Safety Authority; ELISA: Enzyme-linked immunosorbent assay; EE: Ethyl ester; En: Energy; EPA: Eicosapentaenoic acid; GPx: Glutathione peroxidase; HDL: High density lipoprotein; HGH: Human growth hormone; 4-HNE: 4-hydroxy-2,3-transnonenal; HPA: Hypothalamo-pituitary-adrenal axis; HPT: Hypothalamopituitary-testicular axis; HRP: Horseradish peroxidase; IBD: Inflammatory bowel disease; IGF-1: Insulin-like growth factor; IL: Interleukin; LARN: Livelli di Assunzione di Riferimento di Nutrienti ed energia per la popolazione italiana; LDH: Lactate dehydrogenase; LPO: Lipoperoxidation; LMNEF: Low-molecularweight non enzymatic fraction; MDA: Malondialdeyde; MHC: Major histocompatibility complex; MLSS: Maximal lactate steady state; N: Sample size; NDA: EFSA Panel on Dietetic Products, Nutrition and Allergies; 8OHdG: 8-hydroxy-2'-deoxyguanosine; OTS: Overtraining syndrome; PCA: Principal component analysis; PC: Principal components; PCc: Protein Carbonyl content; PPAR- $\rangle$ : Peroxisome proliferator-activated receptor; PRI: Population reference intakes; PUFAs: Polyunsatured fatty acids; RBC: Red blood cells; RDA: Recommended Daily Allowance; RI: Reference Intake range for macronutrients; ROS: Reactive oxygen species; SD: Standard deviation; SED: Sedentary subjects; SINU: Società Italiana di Nutrizione Umana; SOD: Speroxide dismutase; S.r.l.: Società a responsabilità limitata; T: Testosterone; TNF-a: Tumor Necrosis Factor-a; trigly: triglyceride; urn: urine; WHO: World Health Organization

\section{Acknowledgments}

The authors would like to thank all voluntary subjects for their substantial contribution to this study, the athletic Society ATL. VIRTUS CR LUCCA, (Italy), Ethic Sport Italia S.r.l. viale Empoli, 33-47838 Riccione (RN), Italy for providing the fish oil soft capsules of n-3 fatty acids used for the procedures of the pilot study, and Synlab ${ }^{\star}$ Pavia (Italy) for the analysis of some hematological parameters.

\section{Authors' contributions}

Conceptualization, D.B. and A.G.; methodology, D.B.; software, D.B.; validation, M.V.; formal analysis, M.G. and E.D.; investigation, A.G. and D.B.; resources, A.G. and M.V.; data curation, D.B. and M.G.; writing - original draft preparation, D.B.; writing—review and editing, M.V.; visualization, D.B.; supervision, M.D.; project administration, D.B. and M.D. The author(s) read and approved the final manuscript.

\section{Funding}

This research received no external funding.

\section{Availability of data and materials}

The datasets used and/or analyzed during the current study and not shown in the paper are available from the corresponding author on reasonable request.

\section{Ethics approval and consent to participate}

all subjects were fully informed about experimental protocols in which the investigations were carried out following the guidelines of the Declaration of Helsinki of 1975 (https://www.wma.net/what-we-do/medical-ethics/ declaration-of-helsinki/), revised in Tokyo 2004 and subsequently in 2013. All participants approved and signed an informed consent document, authorizing the experimenter to use the results for scientific publication purposes, before enrolling in the study. The Institutional Review Board of the University of Pavia (Italy) approved the study.

\section{Consent for publication}

Not applicable.

Competing interests

The Authors declare that they have no competing interests. 


\section{Author details}

"Department of Biology and Biotechnology "Lazzaro Spallanzani", University of Pavia, Via Ferrata, 9-27100 Pavia (PV), Italy. 'Department of Earth and Environmental Sciences (DSTA) - Unit of Statistical Analyses (UNISTAT)، University of Pavia, Via Ferrata, 9-27100 Pavia (PV), Italy.

Received: 17 February 2020 Accepted: 4 November 2020

Published online: 11 November 2020

\section{References}

1. Billat $V L$, Sirvent P, Py G, Koralsztei JP, Mercier J. The concept of maximal lactate steady state: a bridge between biochemistry, physiology and sport science. Sports Med. 2003;33:407-26.

2. Finkel T. Reactive oxygen species and signal transduction. IUBMB Life. 2001; 52:3-6.

3. Altan O, Pabuçcuoğlu A, Altan A, Konyalioğlu S, Bayraktar H. Effect of heat stress on oxidative stress, lipid peroxidation and some stress parameters in broilers. Br Poult Sci. 2003;44:545-50.

4. Ferreira LF, Reid MB. Muscle-derived ROS and thiol regulation in muscle fatigue. J Appl Physiol. 2008;104:853-60.

5. Filaire E, Massart A, Portier H, Rouveix M, Rosado F, Bage AS, et al. Effect of 6 weeks of n-3 fatty-acid supplementation on oxidative stress in judo athletes. Int J Sport Nutr Exerc Metab. 2010;20:496-506.

6. Kreher JB, Schwartz JB. Overtraining syndrome: a practical guide. Sports Health. 2012;4:128-38.

7. Chung HY, Cesari M, Anton S, Marzetti E, Giovannini S, Seo AY, et al. Molecular inflammation: underpinnings of aging and age-related diseases. Ageing Res Rev. 2009:8:18-30.

8. Lee JY, Sohn KH, Rhee SH, Hwang D. Saturated fatty acids, but not unsaturated fatty acids, induce the expression of cyclooxygenase-2 through toll-like receptor. J Biol Chem. 2001;276:16683-9.

9. Colas RA, Shinohara M, Dalli J, Chiang N, Serhan CN. Identification and signature profiles for pro-resolving and inflammatory lipid mediators in human tissue. Am J Physiol Cell Physiol. 2014;307:C39-54.

10. Serhan CN, Dalli J, Colas RA, Winkler JW, Chiang N. Protectins and maresins: new pro-resolving families of mediators in acute inflammation and resolution bioactive metabolome. Biochim Biophys Acta. 1851;2015:397-413.

11. Mickleborough TD. A nutritional approach to managing exercise-induced asthma. Exerc Sport Sci Rev. 2008:36:135-44.

12. Calder PC. Omega-3 polyunsaturated fatty acids and inflammatory processes: nutrition or pharmacology? Br J Clin Pharmacol. 2013;75:645-62.

13. Simopoulos AP. Omega-3 fatty acids and athletics. Curr Sports Med Rep. 2007;6:230-6.

14. Gray P, Chappell A, Jenkinson AM, Thies F, Gray SR. Fish oil supplementation reduces markers of oxidative stress but not muscle soreness after eccentric exercise. Int J Sport Nutr Exerc Metab. 2014;24:206-14.

15. Rawson ES, Miles MP, Larson-Meyer DE. Dietary supplements for health, adaptation, and recovery in athletes. Int J Sport Nutr Exerc Metab. 2018;28: $188-99$

16. Mickleborough TD. Omega-3 polyunsaturated fatty acids in physical performance optimization. Int J Sport Nutr Exerc Metab. 2013;23:83-96.

17. Da Boit M, Hunter AM, Gray SR. Fit with good fat? The role of $n-3$ polyunsaturated fatty acids on exercise performance. Metabolism. 2017;66: 45-54.

18. Blasbalg TL, Hibbeln JR, Ramsden CE, Majchrzak SF, Rawlings RR. Changes in consumption of omega-3 and omega- 6 fatty acids in the United States during the 20th century. Am J Clin Nutr. 2011;93:950-62.

19. Molodecky NA, Soon IS, Rabi DM, Ghali WA, Ferris M, Chernoff G, et al. Increasing incidence and prevalence of the inflammatory bowel diseases with time, based on systematic review. Gastroenterology. 2012;142:46-54.

20. Goldman DW, Pickett WC, Goetzl EJ. Human neutrophil chemotactic and degranulating activities of leukotriene B5 (LTB5) derived from eicosapentaenoic acid. Biochem Biophys Res Commun. 1983;117:282-8.

21. LARN. Livelli di Assunzione di Riferimento di Nutrienti ed energia. In: Società Italiana di Nutrizione Umana (SINU); 2014. https://sinu.it/2019/07/09/lipidi/. Accessed 10 Oct 2019

22. Dietary reference values for nutrients: summary report. In: EFSA Supporting Publication. 2017. e15121. https://doi.org/10.2903/sp.efsa.2017.e15121. Accessed 26 Nov 2019.
23. Aranceta J, Pe'rez-Rodrigo C. Recommended dietary reference intakes, nutritional goals and dietary guidelines for fat and fatty acids: a systematic review. Br J Nutr. 2012;107(Suppl 2):8-22.

24. Dietary Reference Intakes for Energy, Carbohydrate, Fiber, Fat, Fatty Acids, Cholesterol, Protein, and Amino Acids. In: The National Academies Press, Washington, DC. 2005. https://doi.org/10.17226/10490. Accessed 9 Jul 2020.

25. McDermott BP, Smith CR, Butts CL, Caldwell AR, Lee EC, Vingren JL, et al. Renal stress and kidney injury biomarkers in response to endurance cycling in the heat with and without ibuprofen. J Sci Med Sport. 2018;21:1180-4.

26. Komi PV, Karlsson J. Skeletal muscle fibre types, enzyme activities and physical performance in young males and females. Acta Physiol Scand. 1978;103:210-8

27. Scarth JP. Modulation of the growth hormone-insulin-like growth factor (GH-IGF) axis by pharmaceutical, nutraceutical and environmental xenobiotics: an emerging role for xenobiotic-metabolizing enzymes and the transcription factors regulating their expression. A review. Xenobiotica. 2006 36:119-218.

28. Cherubini A, Ruggiero C, Polidori MC, Mecocci P. Potential markers of oxidative stress in stroke. Free Radic Biol Med. 2005:39:841-52.

29. Dalle-Donne I, Rossi R, Colombo R, Giustarini D, Milzani A. Biomarkers of oxidative damage in human disease. Clin Chem. 2006:52:601-23.

30. Esterbauer $\mathrm{H}$, Schaur RJ, Zollner $\mathrm{H}$. Chemistry and biochemistry of 4 hydroxynonenal, malonaldehyde and related aldehydes. Free Radic Biol Med. 1991;11:81-128.

31. Richter C. Biophysical consequences of lipid peroxidation in membranes. Chem Phys Lipids. 1987:44:175-89.

32. Erdelmeier I, Gerard-Monnier D, Yadan JC, Chaudiere J. Reactions of Nmethyl-2-phenylindole with malondialdehyde and 4-hydroxyalkenals. Mechanistic aspects of the colorimetric assay of lipid peroxidation. Res Toxicol. 1998:11:1184-94.

33. Grune T, Shringarpure R, Sitte N, Davies K. Age-related changes in protein oxidation and proteolysis in mammalian cells. J Gerontol A Biol Sci Med Sci. 2001;56:B459-67.

34. Levine RL, Garland D, Oliver CN, Amici A, Climent I, Lenz AG, et al. Determination of carbonyl content in oxidatively modified proteins. Methods Enzymol. 1990:186:464-78.

35. Lowry OH, Rosebrough NJ, Farr AL, Randall RJ. Protein measurement with the Folin phenol reagent. J Biol Chem. 1951;193:265-75.

36. Candeias LP, Steenken S. Reaction of $\mathrm{HO} \bullet$ with guanine derivatives in aqueous solution: formation of two different redox-active $\mathrm{OH}$-adduct radicals and their unimolecular transformation reactions. Properties of $\mathrm{G}(-\mathrm{H}) \bullet$. Chemistry. 2000;6:475-84.

37. Shigenaga MK, Ames BN. Assays for 8-hydroxy-2'-deoxyguanosine: a biomarker of in vivo oxidative DNA damage. Free Radic Biol Med. 1991;10: 211-6.

38. Flohe L, Gunzler WA. Assays of glutathione peroxidase. Methods Enzymol. 1984:105:114-21.

39. Flohe L, Otting F. Superoxide dismutase assays. Methods Enzymol. 1984;105: 93-104.

40. Aebi H. Catalase in vitro. Methods Enzymol. 1984;105:121-6.

41. Atsumi T, Iwakura I, Kashiwagi Y, Fujisawa S, Ueha T. Free radical scavenging activity in the nonenzymatic fraction of human saliva: a simple DPPH assay showing the effect of physical exercise. Antioxid Redox Signal. 1999;1:53746.

42. Luger A, Deuster PA, Kyle SB, Gallucci WT, Montgomery LC, Gold PW, et al. Acute hypothalamic pituitary-adrenal responses to the stress of treadmill exercise-physiological adaptations to physical-training. N Engl J Med. 1987; 316:1309-15.

43. Florini JR. Hormonal-control of muscle growth. Muscle Nerve. 1987;10:57798.

44. Duclos M. A critical assessment of hormonal methods used in monitoring training status in athletes. Int J Sports Med. 2008:9:56-66.

45. Vining RF, McGinley RA, Maksvytis JJ, Ho KY. Salivary cortisol: a better measure of adrenal cortical function than serum cortisol. Ann Clin Biochem. 1983;20:329-35

46. Wood P. Salivary steroid assays -research or routine? Ann Clin Biochem. 2009;46:183-96.

47. Arango Duque G, Descoteaux A. Macrophage cytokines: involvement in immunity and infectious diseases. Front Immunol. 2014;5:491.

48. Adams DC, Collyer ML. A general framework for the analysis of phenotypic trajectories in evolutionary studies. Evolution. 2009;63:1143-54. 
49. R Core Team. A language and environment for statistical computing. In: R Foundation for Statistical Computing, Vienna, Austria; 2015. https://www.Rproject.org/. Accessed 24 May 2019.

50. Gatti R, De Palo EF. An update: salivary hormones and physical exercise. Scand J Med Sci Sports. 2011;21:157-69.

51. Adlercreutz $\mathrm{H}$, Harkonen M, Kuoppasalmi K, Nãveri H, Huhtaniemi I, Tikkanen $\mathrm{H}$, et al. Effect of training on plasma anabolic and catabolic steroid hormones and their response during physical exercise. Int J Sports Med. 1986;7:27-8.

52. de Souza Vale RG, de Oliveira RD, Pernambuco CS. da Silveira Fontenele de Meneses YP, de Meneses SF, da Silva Novaes J, et al. effects of muscle strength and aerobic training on basal serum levels of IGF-1 and cortisol in elderly women. Arch Gerontol Geriatr. 2009;49:343-7.

53. Timon R, Olcina G, Tomas-Carus P, Munoz D, Toribio F, Raimundo A, et al. Urinary steroid profile after the completion of concentric and concentric/eccentric trials with the same total workload. J Physiol Biochem. 2009:65:105-12.

54. Aquilani R, Brugnatelli S, Dossena M, Maestri R, Delfanti S, Buonocore D, Boschi F, Simeti E, Condino AM, Verri M. Oxaliplatin-Fluoropyrimidine combination (XELOX) therapy does not affect plasma amino acid levels and plasma markers of oxidative stress in colorectal Cancer surgery patients: a pilot study. Nutrients. 2019;11:2667.

55. Rattan SI. Hormesis in aging. Ageing Res Rev. 2008;7:63-78.

\section{Publisher's Note}

Springer Nature remains neutral with regard to jurisdictional claims in published maps and institutional affiliations.

Ready to submit your research? Choose BMC and benefit from:

- fast, convenient online submission

- thorough peer review by experienced researchers in your field

- rapid publication on acceptance

- support for research data, including large and complex data types

- gold Open Access which fosters wider collaboration and increased citations

- maximum visibility for your research: over $100 \mathrm{M}$ website views per year

At BMC, research is always in progress.

Learn more biomedcentral.com/submissions 